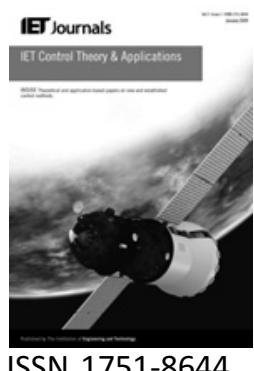

\title{
Sensor fault-tolerant vector control of induction motors
}

\author{
M.E. Romero ${ }^{1}$ M.M. Seron ${ }^{2}$ J.A. De Doná \\ ${ }^{1}$ Laboratorio de Sistemas Dinámicos y Procesamiento de la Información, Universidad Nacional de Rosario, Riobamba 245 bis, \\ 2000 Rosario, Argentina \\ ${ }^{2}$ Centre for Complex Dynamic Systems and Control, The University of Newcastle, Callaghan NSW 2308, Australia \\ E-mail: maria.seron@newcastle.edu.au
}

\begin{abstract}
The authors propose a multisensor switching strategy for fault-tolerant vector control of induction motors. The proposed strategy combines three current sensors and associated observers that estimate the rotor flux. The estimates provided by the observers are compared at each sampling time by a switching mechanism which selects the sensors-observer pair with the smallest error between the estimated flux magnitude and a desired flux reference. The estimates provided by the selected pair are used to implement a vector control law. The authors consider both field-oriented control and direct torque and flux control schemes. Pre-checkable conditions are derived that guarantee fault tolerance under an abrupt fault of a current sensor. These conditions are such that the observers that use measurements from the faulty sensor are automatically avoided by the switching mechanism, thus maintaining good performance levels even in the presence of a faulty sensor. Simulation results under realistic conditions illustrate the effectiveness of the scheme.
\end{abstract}

\section{Introduction}

In recent years, a substantial research interest in multisensor schemes has accompanied the increasing availability of a variety of sensors in many application areas. Multisensor schemes combine data supplied by different sensors to provide more accurate and reliable information (see e.g. [1]). Moreover, the redundancy provided by multiple sensors can be exploited to achieve robustness against malfunctioning or faults in some of the sensors. This faulttolerant idea has been recently explored by the authors in multisensor switching strategies for feedback control systems $[2,3]$. In the current paper, we investigate the use of similar ideas for vector control of induction motors. The use of induction motor drives in high-performance servo systems, such as automated production or transportation systems, has become increasingly popular owing to recent advances in motor control techniques [4]. In such applications, faults in any of the components of the feedback loop (the motor itself, the driving circuits, sensors, actuators etc.) can seriously deteriorate the overall system performance [5]. It is thus crucial, in order to avoid failure of the whole system, to design control strategies that are 'fault tolerant', that is, strategies that are capable to maintain stability and good performance levels even under the occurrence of severe component faults.

In this paper, we propose the use of a sensor fault-tolerant control strategy that combines stator current sensors, rotor flux observers and vector control of the induction motor, as illustrated in Fig. 1. More precisely, the scheme consists of a bank of three rotor flux observers, an estimate switching mechanism and the vector controller. Each observer provides estimates of the rotor flux based on noisy measurements of two-phase currents. At each sampling time, the switching mechanism selects the sensors-observer pair with the smallest error between the estimated rotor flux magnitude and a desired flux reference, and passes the selected state estimates to the vector controller. We consider two popular vector control techniques, namely, field-oriented control (FOC) $[6,7]$ and direct torque and flux control (DTFC) $[8,9]$. The implementation of the FOC technique requires measurements or estimates of the rotor speed, stator currents and rotor flux. We will assume here that the rotor speed and stator currents are measured. In contrast, the rotor flux is estimated from the available 


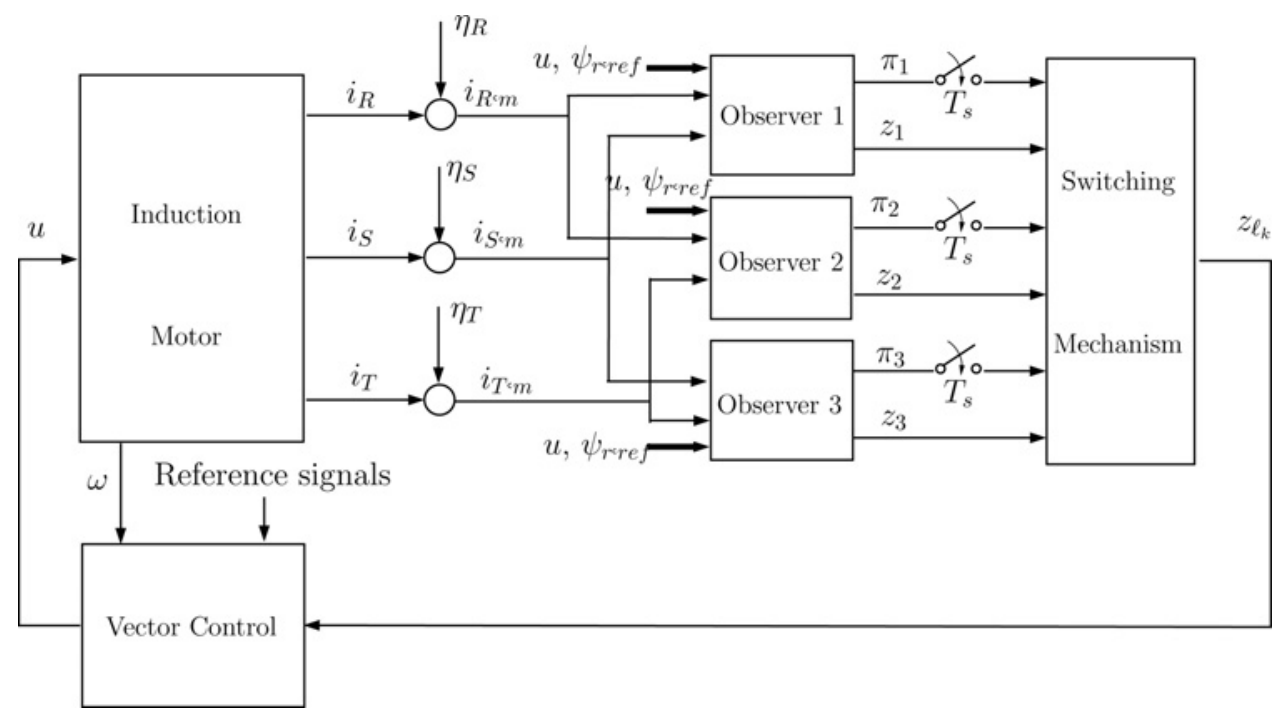

Figure 1 Fault-tolerant control scheme for the induction motor

measurements by means of flux observers of the form proposed Kubota and Matsuse [10]. On the other hand, the DTFC technique requires measurements or estimates of the stator current and stator flux for the calculation of the electromagnetic torque. In this paper, the stator flux is estimated from the available measurements by means of a full-order Luenberger-type observer.

A standard approach to achieve fault tolerance is to equip the control system with explicit fault detection and compensation capabilities; see, for example, [5, 11-15] for the use of this approach in induction motor control systems. In [5], three current sensors are employed and the mechanism used for the detection of sensor faults is the current balance condition, that is, the condition that the sum of the three-phase current is equal to zero. The diagnosis mechanism involves two stages, which means that sensor faults in one phase can be instantaneously detected but not in the others. This characteristic may produce some transients in the regulated variables. The scheme proposed in [12] was pioneer in treating induction motor controller performance together with controller fault tolerance. Indeed, the system developed in [12] adaptively changes control techniques in the event of sensor loss or sensor recovery to attain the best performance given the complement of remaining sensors. Fault detection is achieved by means of a signature detection scheme. The work in [13] proposes a reconfigurable control scheme that also switches among different control strategies depending on the detected sensor fault (current and speed sensors). The detection mechanism is a fuzzy logic block with different weight functions, which evaluates, and fuses, flux position estimated values provided by different motor models. The selection of the fusion weighting functions in the fuzzy logic block is such that more weight is given to consistent estimates and less weight to estimates that are far away from the consensus of the majority. A similar approach is developed in $[14,15]$ with applications to electric and hybrid vehicle traction. In these works, a decision block changes the control strategies (indirect vector control, sensorless vector control, sensorless scalar control and scalar control) depending on the detected sensor fault (current and speed) and the operation conditions. The authors also present a condition for controller smooth transitions.

In contrast with the above approaches, our scheme achieves fault detection and isolation (FDI) and automatic controller reconfiguration 'implicitly' by guaranteeing that the switching cost avoids selecting faulty sensors. Similar to [5], the proposed scheme requires sensor redundancy by measuring the three stator phase currents instead of only two, as is common practice in industrial electrical drive technology. Although the proposed measurement set-up would be slightly more costly at the construction stage, it is likely to save considerably larger costs in many applications because of potential production losses and prolonged downtimes caused by sensor failures. Overall, the main contributions of the present paper are as follows:

1. To eliminate the need for separate FDI and controller reconfiguration units, which are substituted by a simple estimate switching mechanism. Moreover, the proposed approach is modular since the estimate switching mechanism can be combined with any desired vector control technique.

2. To provide off-line pre-checkable conditions in terms of bounds on sensor noises, motor parameters and operating range, which guarantee that, when a current sensor fails, the observers that use measurements from the faulty sensor are automatically avoided by the switching mechanism, hence maintaining good performance levels even under severe sensor faults.

3. To always maintain the performance levels associated with the chosen vector control strategy, which remains unchanged 
even under sensor fault, a property afforded by the use of sensor redundancy; moreover, the latter is also exploited for the instantaneous detection of faults in any of the three phases.

An aspect of this proposed scheme that should be emphasised is the fact that it endows well-established control techniques for induction motors, namely, FOC and DTFC, with sensor fault-tolerant capabilities. In that sense, it should be appreciated that no theoretical stability proofs exist, in general, for these control techniques when implemented using rotor and stator flux estimates (rather than true values), save for the important fact that they work in numerous real-life implementations in industry. Thus, a pervasive assumption of our approach here will be that the vector controllers effectively stabilise the machine and that permanent regime is reached before the occurrence of any fault. Under these circumstances, as the analysis of this paper shows, the proposed scheme guarantees fault tolerance of the closed-loop system.

The remainder of the paper is organised as follows. Section 2 presents the model of the induction motor. Section 3 briefly explains the principle of vector control techniques. Section 4 describes the sensing scheme and flux observers. Section 5 presents the proposed estimate switching mechanism and controller implementation. Section 6 analyses the closedloop system performance under healthy operation of all sensors. Section 7 analyses the system performance under an abrupt fault of a current sensor and derives conditions for fault tolerance of the scheme. Section 8 describes the application of the proposed fault-tolerant strategy to FOC (Section 8.1) and DTFC (Section 8.3). Sections 8.2 and 8.4 illustrate the results by means of simulation examples. Finally, the paper is concluded in Section 9. Preliminary versions of the current paper were presented in [16] in combination with the FOC technique and in [17] with the DTFC technique. An initial study of extensions to speed sensorless control was communicated in [18].

\section{Model of the induction motor}

The induction motor can be represented in different frames depending on the control scheme to be implemented. For the purposes of the proposed fault-tolerant switching strategy, it will be convenient to consider the model of the induction motor in a reference frame fixed with the stator, with components denoted by $(a, b)$ [19]. In this reference frame, the motor electromagnetic variables can be described by the following dynamic equation (the dependency of the variables on time $t$ is omitted for simplicity of notation)

$$
\begin{aligned}
\frac{\mathrm{d} x}{\mathrm{~d} t} & =A(\omega) x+B u \\
x & =\left[\begin{array}{llll}
i_{\mathrm{s} a} & i_{\mathrm{s} b} & \psi_{\mathrm{r} a} & \psi_{\mathrm{r} b}
\end{array}\right]^{\mathrm{T}} \\
u & =\left[\begin{array}{ll}
u_{\mathrm{s} a} & u_{\mathrm{s} b}
\end{array}\right]^{\mathrm{T}}
\end{aligned}
$$

where the state $x$ is composed of the stator current and rotor flux components and the input $u$ consists of the stator voltage components. The matrices $A(\omega)$ and $B$ in (1) have the form

$$
A(\omega)=\left[\begin{array}{ll}
a_{\mathrm{r} 11} \mathcal{I} & a_{\mathrm{r} 12} \mathcal{I}+a_{i 12}(\omega) \mathcal{J} \\
a_{\mathrm{r} 21} \mathcal{I} & a_{\mathrm{r} 22} \mathcal{I}+a_{i 22}(\omega) \mathcal{J}
\end{array}\right], \quad B=\left[\begin{array}{c}
\frac{1}{\sigma L_{\mathrm{s}}} \mathcal{I} \\
0
\end{array}\right]
$$

where

$$
\begin{gathered}
a_{\mathrm{r} 11} \triangleq-\frac{R_{\mathrm{s}}}{\sigma L_{\mathrm{s}}}-\frac{1-\sigma}{\sigma \tau_{\mathrm{r}}}, \quad a_{\mathrm{r} 12} \triangleq \frac{1}{c \tau_{\mathrm{r}}}, \quad a_{i 12}(\omega) \triangleq-\frac{n_{\mathrm{p}} \omega}{c} \\
a_{\mathrm{r} 21} \triangleq \frac{M}{\tau_{\mathrm{r}}}, \quad a_{\mathrm{r} 22} \triangleq-\frac{1}{\tau_{\mathrm{r}}}, \quad a_{i 22}(\omega) \triangleq n_{\mathrm{p}} \omega \\
\mathcal{I}=\left[\begin{array}{ll}
1 & 0 \\
0 & 1
\end{array}\right], \quad \mathcal{J}=\left[\begin{array}{cc}
0 & -1 \\
1 & 0
\end{array}\right]
\end{gathered}
$$

and where $n_{\mathrm{p}}$ is the number of pole pairs of the induction machine; $R_{\mathrm{s}}, R_{\mathrm{r}}, L_{\mathrm{s}}, L_{\mathrm{r}}$, are the stator and rotor resistances and self-inductances, respectively; $M$ is the mutual inductance, $\quad \tau_{\mathrm{r}}=L_{\mathrm{r}} / R_{\mathrm{r}}, \quad \sigma=1-M^{2} /\left(L_{\mathrm{s}} L_{\mathrm{r}}\right) \quad$ and $c=\sigma L_{\mathrm{s}} L_{\mathrm{r}} / M$. In addition, the rotor speed $\omega$ satisfies the dynamic equation

$$
\frac{\mathrm{d} \omega}{\mathrm{d} t}=\frac{n_{\mathrm{p}} M}{J L_{\mathrm{r}}}\left[\psi_{\mathrm{r} a} i_{\mathrm{s} b}-\psi_{\mathrm{r} b} i_{\mathrm{s} a}\right]-\frac{\tau_{\mathrm{L}}}{J}
$$

where $J$ is the moment of inertia of the rotor and $\tau_{\mathrm{L}}$ is the load torque, which is assumed constant. For future reference, we define the magnitude of the rotor flux as (see also Appendix 1)

$$
\psi_{\mathrm{r} d}=\sqrt{\psi_{\mathrm{r} a}^{2}+\psi_{\mathrm{r} b}^{2}}
$$

We observe that the stator current components $i_{\mathrm{s} a}, i_{\mathrm{s} b}$ in (1) are the two-phase projection on the $(a, b)$ plane of the threephase currents $i_{\mathrm{R}}, i_{\mathrm{S}}$ and $i_{\mathrm{T}}$. This projection is given by the transformation [19]

$$
\begin{aligned}
& i_{\mathrm{s} a}=\frac{1}{3}\left(2 i_{R}-i_{S}-i_{T}\right) \\
& i_{\mathrm{s} b}=\frac{\sqrt{3}}{3}\left(i_{S}-i_{T}\right)
\end{aligned}
$$

To implement the control law, some of the induction motor states will be assumed to be measured and others will be estimated via state observers. In particular, we will assume that the rotor speed $w$ and the phase currents $i_{\mathrm{R}}, i_{\mathrm{S}}$ and $i_{\mathrm{T}}$ are measured. The equations in (8) then directly give the state variables $i_{\mathrm{s} a}$ and $i_{\mathrm{s} b}$. The remaining state variables $\psi_{\mathrm{r} a}$ and $\psi_{\mathrm{r} b}$ in (1) will be estimated by means of flux observers. 


\section{Vector control schemes}

Vector control (VC) of induction motors (which involves vectorial representation of the electromagnetic variables) is now a current methodology popularised by its two main commercial implementations: FOC and DTFC. When compared with scalar control (which involves scalar representation of the motor electromagnetic variables), the VC technique has superior transient response, although at the expense of an extra amount of calculation time as well as the need of current measurements. Besides the mentioned schemes, FOC and DTFC, many different VC approaches can be found in the literature which use advanced tools of control theory such as exact feedback linearisation, sliding mode control, model predictive control, output regulation subspace control etc. (see, e.g. [7, 20-23]).

The main characteristic of $\mathrm{VC}$ is the possibility to control the (rotor or stator) flux magnitude, on the one hand, and rotor velocity or electromagnetic torque, on the other hand, with two different control loops. Thus, the VC approach allows us to view and control the induction motor in a similar way to a direct-current motor (especially in FOC where both control loops are decoupled). More specifically, VC methods propose two control objectives (and associated controlled outputs) that typically consist of (i) regulation of stator or rotor flux magnitude to desired reference values $\psi_{\mathrm{s} \text {,ref }}$ or $\psi_{\mathrm{r} \text {,ref }}$, respectively and (ii) regulation of rotor velocity or electric torque to desired reference values $\omega_{\text {ref }}$ or $\tau_{\text {ref }}$, respectively. Based on these two objectives, we can express the vector control law, in a generalised form, as a function of the rotor speed $\omega$, the state $x$ of system (1), the stator flux components $\psi_{\mathrm{s} a}, \psi_{\mathrm{s} b}$, and the aforementioned reference signals, that is

$$
u=\mathcal{K}_{\mathrm{VC}}\left(\omega, x, \psi_{\mathrm{s} a}, \psi_{\mathrm{s} b}, \psi_{\mathrm{s}, \mathrm{ref}}, \psi_{\mathrm{r}, \mathrm{ref}}, \omega_{\mathrm{ref}}, \tau_{\mathrm{ref}}\right)
$$

where the specific form of the function $\mathcal{K}_{\mathrm{VC}}$ depends on the particular VC scheme selected and will become clear in Section 8 (in particular, $\mathcal{K}_{\mathrm{VC}}$ does not depend on $\psi_{\mathrm{s} \text {,ref }}$ and $\tau_{\text {ref }}$ in FOC, and it does not depend on $\psi_{\mathrm{r}, \text { ref }}$ in DTFC). In Sections 4 and 5 below, we will describe a strategy for measurement and estimation of the states required to implement the VC law (9).

\section{Current sensors and flux observers}

As mentioned in Section 2, the phase currents $i_{\mathrm{R}}, i_{\mathrm{S}}$ and $i_{\mathrm{T}}$ are available for measurement. These currents satisfy the algebraic relation

$$
i_{\mathrm{R}}+i_{\mathrm{S}}+i_{\mathrm{T}}=0
$$

Hence, if two-phase currents are measured then the thirdphase current can be calculated from (10). However, we propose to employ three sensors measuring the three currents $i_{\mathrm{R}}, i_{\mathrm{S}}$ and $i_{\mathrm{T}}$, and take advantage of the redundancy provided by these three measurements in an observer-based strategy for fault-tolerant control as described below.

The sensor measurement equations have the form

$$
\begin{gathered}
i_{R, m}=i_{R}+\eta_{R, \mathrm{~h}} \\
i_{S, m}=i_{S}+\eta_{S, \mathrm{~h}} \\
i_{T, m}=i_{T}+\eta_{T, \mathrm{~h}}
\end{gathered}
$$

where $\eta_{R, \mathrm{~h}}, \eta_{S, \mathrm{~h}}$ and $\eta_{T, \mathrm{~h}}$ are bounded, zero-mean ('healthy') measurement noises.

Remark 1: The approach followed in this paper is purely deterministic and does not require any specific statistical description for the sensor noises. The 'zero-mean' assumption is not restrictive and is made so that the resulting 'mean value' can be taken to be zero after the noise is low-pass filtered (see Section 5). Thus, the measurement noises can be considered as random variables but we do not assume knowledge of their statistical properties except for their mean value; however, we require the knowledge of their bounds. Hence, noises with uniform distribution or any truncated distribution, for example, are within the scope of our analysis.

For the fault-tolerant switching strategy, we compute the rotor flux components independently of the vector control method to be used. To this end, we employ three observers, each one based on measurements from two phases. That is, the $j$ th observer, for $j=1,2,3$, uses its associated phase currents $i_{\mathrm{R}, j}, i_{\mathrm{S}, j}$ and $i_{\mathrm{T}, j}$ given by the following equations based on the measured currents $(11)-(13)$ and $(10)$

$$
\begin{aligned}
& i_{R, 1}=i_{R, m}, \quad i_{S, 1}=i_{S, m}, \quad i_{T, 1}=-i_{R, m}-i_{S, m} \\
& i_{R, 2}=i_{R, m}, \quad i_{S, 2}=-i_{R, m}-i_{T, m}, \quad i_{T, 2}=i_{T, m} \\
& i_{R, 3}=-i_{S, m}-i_{T, m}, \quad i_{S, 3}=i_{S, m}, \quad i_{T, 3}=i_{T, m}
\end{aligned}
$$

Then, the associated $(a, b)$ projections for $j=1,2,3$ are computed using (8) as

$i_{\mathrm{s} a, j}=\frac{1}{3}\left(2 i_{R, j}-i_{S, j}-i_{T, j}\right), \quad i_{\mathrm{s} b, j}=\frac{\sqrt{3}}{3}\left(i_{S, j}-i_{T, j}\right)$

Finally, the values of $i_{s a, j}$ and $i_{s b, j}$ obtained in (17) are used in the observer dynamic equation

$$
\frac{\mathrm{d} \hat{x}_{j}}{\mathrm{~d} t}=A(\omega) \hat{x}_{j}+B u+G_{K}(\omega)\left[\begin{array}{l}
\hat{i}_{\mathrm{s} a, j}-i_{\mathrm{s} a, j} \\
\hat{i}_{\mathrm{s} b, j}-i_{\mathrm{s} b, j}
\end{array}\right], \quad j=1,2,3
$$

where $\hat{x}_{j}=\left[\hat{i}_{\mathrm{s} a, j} \hat{i}_{\mathrm{s} b, j} \hat{\psi}_{\mathrm{r} a, j} \hat{\psi}_{\mathrm{r} b, j}\right]^{\mathrm{T}}$ is the state estimate provided by the $j$ th observer, and $A(\omega), B$ and $u$ are as in 
(1). The observer gain matrix $G_{K}(\omega)$ in (18) has the following form proposed by [10]

$$
G_{K}(\omega)^{\mathrm{T}}=\left[\begin{array}{cccc}
g_{1} & g_{2}(\omega) & g_{3} & g_{4}(\omega) \\
-g_{2}(\omega) & g_{1} & -g_{4}(\omega) & g_{3}
\end{array}\right]
$$

where, given a scalar parameter $K>1, g_{1}=(K-1)$ $\left(a_{r 11}+a_{r 22}\right), g_{2}(\omega)=(K-1) a_{i 22}(\omega), g_{3}=\left(K^{2}-1\right)\left(a_{r 21}+\right.$ $\left.a_{r 11} c\right)-c g_{1}, g_{4}(\omega)=-c g_{2}(\omega)$, and all remaining symbols are as defined in (3)-(5). This gain matrix is such that for each fixed value of $\omega$, the eigenvalues of the observer closed-loop matrix $A(\omega)+G_{K}(\omega) C$, with

$$
C=\left[\begin{array}{llll}
1 & 0 & 0 & 0 \\
0 & 1 & 0 & 0
\end{array}\right]
$$

have negative real part and are proportional to those of $A(\omega)$ by a factor of $K$. That is

$$
\operatorname{eig}\left(A(\omega)+G_{K}(\omega) C\right)=K \operatorname{eig} A(\omega)
$$

The following output equation is associated with the $j$ th observer, for $j=1,2,3$

$$
z_{j}=\left[\begin{array}{llll}
i_{s a, j} & i_{\mathrm{s} b, j} & \hat{\psi}_{\mathrm{r} a, j} & \hat{\psi}_{\mathrm{r} b, j}
\end{array}\right]^{\mathrm{T}}
$$

The variable $z_{j}$ in (22) is the signal that the $j$ th observer will make available to the controller whenever the switching mechanism selects this observer to implement the control law. Note that $z_{j}$ contains the measured values of $i_{\mathrm{sa}, j}$ and $i_{\mathrm{sb}, j}$ (as is standard in, e.g., sensorless FOC, see e.g. [10]). An alternative is to use the estimated values $\hat{i}_{s a, j}, \hat{i}_{s b, j}$, which are filtered versions of the noisy measurements of these variables [24].

In the following section, we will describe a mechanism to switch between the above three observers according to a performance selection criterion. The observer that achieves the best value of the criterion will pass its output $\left(z_{1}, z_{2}\right.$ or $z_{3}$ ) to be used in the implementation of the VC law (9).

Remark 2: As we will see in Section 8.1, in the case of FOC, when an observer is selected by the switching mechanism, all elements of its associated output $z_{j}, j \in\{1$, $2,3\}$, are used to implement the control law. On the other hand, as explained in Section 8.3, in the case of DTFC only the currents $i_{s a, j}, i_{s b, j}$ (which can be computed as $C z_{j}$, with $C$ as in (20)) are used to estimate stator flux and electromagnetic torque by means of an additional observer.

\section{Estimate switching and control implementation}

When the induction motor reaches a permanent regime, the squared magnitude of the rotor flux, $\psi_{\mathrm{r} d}^{2} \triangleq \psi_{\mathrm{r} a}^{2}+\psi_{\mathrm{r} b}^{2}$ is (ideally) equal to its desired constant reference squared value $\psi_{\mathrm{r} \text {,ref }}^{2}$ (see Appendix 1 for an analysis of the induction motor variables in permanent regime). Thus, we could assess each observer's performance by considering the error signal $\hat{\psi}_{\mathrm{r} d, j}^{2}-\psi_{\mathrm{r}, \mathrm{ref}}^{2}$, where $\hat{\psi}_{\mathrm{r} d, j}^{2} \triangleq \hat{\psi}_{\mathrm{r} a, j}^{2}+\hat{\psi}_{\mathrm{r} b, j}^{2}, j=1,2,3$, is the estimated squared magnitude of the rotor flux. (This difference can be regarded as, what is commonly known as, the 'estimation tracking error'; that is, the 'tracking error' of the estimation with respect to its reference value.) However, as is well known, the input voltages of an induction motor are PWM signals generated by the inverter that actuates the motor. These PWM signals have a fundamental component with appropriate amplitude and frequency that drives the motor to the permanent regime. The higher harmonic components, on the other hand, lead to the, so-called, 'ripple' (or high-frequency oscillation) of the electromagnetic variables, which is usually attenuated by the 'low-pass' nature of the induction motor itself. Hence, the actual magnitude of the rotor flux can be seen as the sum of a mean value and a (small) high-frequency ripple. We thus propose to filter the signal $\hat{\psi}_{\mathrm{r} d, j}^{2}$ before computing the error signal. That is, for each observer we consider the following 'error signal' or 'residual'

$$
\pi_{j} \triangleq\left|\overline{\hat{\psi}_{\mathrm{rd}, j}^{2}}-\psi_{\mathrm{r}, \mathrm{ref}}^{2}\right|, \quad j=1,2,3
$$

which measures the deviation of the filtered estimated squared magnitude of the rotor flux, denoted as $\hat{\psi}_{\mathrm{rd},}^{2}$, , from the rotor flux squared reference signal, denoted as $\psi_{\mathrm{r} \text {,ref }}^{2}$. The filtered values are obtained by passing each signal through a lowpass filter with transfer function

$$
H(s)=\frac{1}{\left(T_{\mathrm{H}^{s}}+1\right)^{2}}
$$

The design parameter $T_{\mathrm{H}}>0$ in (24) will be chosen so that all harmonic components of the signals are sufficiently attenuated and the resulting filtered signals essentially represent their mean values.

Remark 3: We observe that other expressions for the residual signal, instead of (23), can be used by the switching mechanism to perform the estimate selection. However, the choice (23) for different vector control schemes is convenient because of the independence of the rotor flux magnitude from the load torque, which, in turn, reduces the sensitivity of the switching mechanism to changes in the load state of the induction motor. Note also that, although the stator current measurements are immediately affected by a sensor fault, it is not immediate to construct a residual using these measurements since the 'reference' value for those currents strongly depends on the machine's operating and load conditions as opposed to the rotor flux reference which is given externally. 
The residual signals $\pi_{j}$ are subsequently sampled with a sampling period $T_{\mathrm{s}}$ to obtain the discrete-time signals

$$
\pi_{j}[k]=\pi_{j}\left(k T_{\mathrm{s}}\right), \quad k=0,1, \ldots
$$

for $j=1,2,3$. Finally, the sampled residual signals $\pi_{j}[k]$ are compared at each sampling time $k$ according to the following switching criterion

$$
\ell_{k}=\operatorname{argmin}_{j}\left\{\pi_{j}[k]: j \in\{1,2,3\}\right\}
$$

That is, $\ell_{k}$ in (26) is the index of the observer whose sampled residual signal at time $k, \pi_{\ell_{k}}[k]$, is the smallest among all three observers.

At each sampling time $k$, then, the observer with index $\ell_{k}$ computed from (26) is selected by the switching mechanism and its output $z_{\ell_{k}}\left(k T_{\mathrm{s}}\right)$ passed on to the controller during $k T_{\mathrm{s}} \leq t<(k+1) T_{\mathrm{s}}$ to substitute for the real state $x$ in the implementation of the VC law (9). Note that the switching is performed in software, that is, there are no movable parts nor switching electronics involved. In addition, the variables being switched, $z_{1}, z_{2}$ or $z_{3}$, in normal operation, take very similar values, hence the switching can be regarded as 'benign' for the system operation.

For those VC methodologies, such as DTFC, which also require the stator flux components for their implementation, an additional observer [driven by the current measurements selected by the switching mechanism, that is, $C z_{\ell_{k}}\left(k T_{\mathrm{s}}\right)$, with $C$ as in (20), see Remark 2] will be employed to provide estimates $\hat{\psi}_{s a}, \hat{\psi}_{s b}$ of these components (see Section 8.3). Thus, the controller is implemented in the following way

$$
\begin{aligned}
u(t)= & \mathcal{K}_{\mathrm{VC}}\left(\omega\left(k T_{\mathrm{s}}\right), z_{\ell_{k}}\left(k T_{\mathrm{s}}\right), \hat{\psi}_{\mathrm{s} a}\left(k T_{\mathrm{s}}\right), \hat{\psi}_{\mathrm{s} b}\left(k T_{\mathrm{s}}\right), \psi_{\mathrm{s}, \mathrm{ref}}, \psi_{\mathrm{r}, \mathrm{ref}},\right. \\
& \left.\omega_{\mathrm{ref}}, \tau_{\mathrm{ref}}\right), \quad t \in\left[k T_{\mathrm{s}},(k+1) T_{\mathrm{s}}\right)
\end{aligned}
$$

When compared with standard vector control strategies based on the use of a single observer, the proposed multi-observer switching strategy has similar performance under healthy operation of all current sensors and, more importantly, it has the advantage of preserving good performance levels under sensor outage. These properties will be analysed in the following sections and illustrated by simulation tests in Sections 8.2 and 8.4 .

\section{Performance under healthy operation}

In this section we will analyse the performance of the scheme of Fig. 1 in permanent regime and when all current sensors are operational. (As stated in Section 1, a pervasive assumption of our approach is that the vector controllers - which have here been adopted from the literature - effectively stabilise the machine and that permanent regime is reached before the occurrence of any fault.)

\subsection{Induction motor variables}

In permanent regime the rotor speed $\omega$ and the flux magnitude $\psi_{\mathrm{r} d}$ in (7) reach the desired constant values $\omega=\omega_{\text {ref }}$ and $\psi_{\mathrm{r} d}=\psi_{\mathrm{r}, \mathrm{ref}}$. The permanent regime values of the flux and current components are as computed in Appendix 1. Those expressions for the induction motor variables in permanent regime will be used in Section 7 to analyse the performance of the multisensor switching system under faulty operation and to derive fault tolerance conditions.

\subsection{Observer variables}

To analyse the observer variables in permanent regime, we define the estimation errors as

$$
\tilde{x}_{j}=\left[\begin{array}{llll}
\tilde{i}_{\mathrm{s} a, j} & \tilde{i}_{\mathrm{s} b, j} & \tilde{\psi}_{\mathrm{ra} a, j} & \tilde{\psi}_{\mathrm{r} b, j}
\end{array}\right]^{\mathrm{T}} \triangleq x-\hat{x}_{j}, \quad j=1,2,3
$$

where $x$ is the state of the system (1) and $\hat{x}_{j}$ is the state estimate provided by observer $j$, for $j=1,2,3$. It is easy to show using (1), (20) and the observer equations (14), (17) and (18) that, under healthy operation of all current sensors, the estimation errors (28) satisfy the dynamic equations

$$
\dot{\tilde{x}}_{j}=\left[A(\omega)+G_{K}(\omega) C\right] \tilde{x}_{j}+G_{K}(\omega) \eta_{j}, \quad j=1,2,3
$$

where, for each fixed $\omega$, the matrix $A(\omega)+G_{K}(\omega) C$ is stable by design (see discussion after (19), in particular (21)) and $\eta_{1}=\left[\begin{array}{ll}\eta_{R, \mathrm{~h}} & (\sqrt{3} / 3)\left(\eta_{R, \mathrm{~h}}+2 \eta_{S, \mathrm{~h}}\right)\end{array}\right]^{\mathrm{T}}, \eta_{2}=\left[\eta_{R, \mathrm{~h}}\right.$ $\left.-(\sqrt{3} / 3)\left(\eta_{R, \mathrm{~h}}+2 \eta_{T, \mathrm{~h}}\right)\right]^{\mathrm{T}} \quad$ and $\quad \eta_{3}=\left[-\left(\eta_{S, \mathrm{~h}}+\eta_{T, \mathrm{~h}}\right)\right.$ $\left.-(\sqrt{3} / 3)\left(\eta_{S, \mathrm{~h}}+\eta_{T, \mathrm{~h}}\right)\right]^{\mathrm{T}}$.

When $\omega=\omega_{\text {ref }}$ in permanent regime, since the noises $\eta_{j}$, $j=1,2,3$ are bounded by assumption, the states of (29) will be ultimately bounded. In particular, using a straightforward modification of Theorem 1 in [25], we can obtain the following result that derives ultimate bounds on the flux estimation errors. (The notations $|M|$ and $\mathbb{R e}(M)$ indicate the elementwise magnitude and real part, respectively, of a (possibly complex) matrix (vector) $M$.)

Lemma 1: Let the noises be elementwise bounded as $\left|\eta_{j}\right| \leq \bar{\eta}_{j}, j=1,2,3$, for some vectors $\bar{\eta}_{j}$ with positive elements. Let $V \Lambda V^{-1}$ be the Jordan decomposition of the matrix $A\left(\omega_{\text {ref }}\right)+G_{K}\left(\omega_{\text {ref }}\right) C$, for constant $\omega_{\text {ref }}$. Then the flux estimation errors are elementwise ultimately bounded as

$$
\begin{aligned}
\left|\tilde{\psi}_{\mathrm{r} a, j}\right| & \leq \epsilon_{a, j} \\
\left|\tilde{\psi}_{\mathrm{r} b, j}\right| & \leq \epsilon_{b, j}
\end{aligned}
$$


for $j=1,2,3$, where

$$
\begin{aligned}
& \epsilon_{a, j} \triangleq\left[\begin{array}{llll}
0 & 0 & 1 & 0
\end{array}\right]|V|\left|\mathbb{R e}(\Lambda)^{-1}\right|\left|V^{-1} G_{K}\left(\omega_{\text {ref }}\right)\right| \bar{\eta}_{j} \\
& \epsilon_{b, j} \triangleq\left[\begin{array}{llll}
0 & 0 & 0 & 1
\end{array}\right]|V|\left|\mathbb{R e}(\Lambda)^{-1}\right|\left|V^{-1} G_{K}\left(\omega_{\text {ref }}\right)\right| \bar{\eta}_{j}
\end{aligned}
$$

Proof: The result follows by direct application of Theorem 1 in [25].

We next use Lemma 1 to obtain ultimate bounds for the observer residual signals (23). First, using the fact that $\psi_{\mathrm{r} d}^{2}=\psi_{\mathrm{r} a}^{2}+\psi_{\mathrm{r} b}^{2}=\psi_{\mathrm{r}, \mathrm{ref}}^{2}$ in permanent regime, we can write

$$
\begin{aligned}
\pi_{j} & =\left|\overline{\hat{\psi}_{\mathrm{r} a, j}^{2}+\hat{\psi}_{\mathrm{r} b, j}^{2}}-\psi_{\mathrm{r}, \mathrm{ref}}^{2}\right| \\
& =\left|\overline{\left(\psi_{\mathrm{r} a}-\tilde{\psi}_{\mathrm{r} a, j}\right)^{2}+\left(\psi_{\mathrm{r} b}-\tilde{\psi}_{\mathrm{r} b, j}\right)^{2}}-\psi_{\mathrm{r}, \mathrm{ref}}^{2}\right| \\
& =\left|\overline{\tilde{\psi}_{\mathrm{r} a, j}^{2}}+\overline{\tilde{\psi}_{\mathrm{r} b, j}^{2}}-2 \overline{\psi_{\mathrm{r} a} \tilde{\psi}_{\mathrm{r} a, j}}-2 \overline{\psi_{\mathrm{r} b} \tilde{\psi}_{\mathrm{r} b, j}}\right|
\end{aligned}
$$

for $j=1,2,3$, where a bar over a signal denotes the signal filtered by the transfer function (24). Thus, using (30) and noting that the transfer function $H(s)$ in (24) has two real poles and is such that $H(0)=1$ (and hence upper bounds on its output signal are less than or equal to upper bounds on its input signal), we have

$$
\pi_{j} \leq \epsilon_{a, j}^{2}+\epsilon_{b, j}^{2}+2 \psi_{\mathrm{r}, \mathrm{ref}} \epsilon_{a, j}+2 \psi_{\mathrm{r}, \mathrm{ref}} \epsilon_{b, j}
$$

Finally, the sampled residual signal $\pi_{j}[k], j=1,2,3$, defined in (25), satisfies the same bounds, that is

$$
\pi_{j}[k] \leq \bar{\pi}_{j}, \quad \bar{\pi}_{j} \triangleq \epsilon_{a, j}^{2}+\epsilon_{b, j}^{2}+2 \psi_{\mathrm{r}, \mathrm{ref}}\left(\epsilon_{a, j}+\epsilon_{b, j}\right)
$$

where $\epsilon_{a, j}$ and $\epsilon_{b, j}$ are defined in (31).

If the bounds on the noises are small, then the bounds (33) on the sampled observer residual signals under healthy operation will also be small. As we will show in Section 7 below, these observer residual signals, when a current sensor associated with the corresponding observer fails, are bounded and - more importantly - separated from the 'healthy' bounded signals (33) [see (44) and (45)]. This 'separation' between healthy and faulty operation is the key to achieve fault tolerance in the proposed approach.

\section{Performance under sensor fault and fault tolerance}

In this section, we analyse the performance of the switching control scheme under abrupt faults of current sensors. We will model an abrupt fault as an instantaneous change in one of the sensor measurement equations, from (11)-(13) to

$$
i_{j, m}=\left(1-\alpha_{j}\right) i_{j}+\eta_{j, \mathrm{f}}, \quad j \in\{R, S, T\}
$$

where $\alpha_{j} \in(0,1], j \in\{R, S, T\}$, and $\eta_{R, \mathrm{f}}, \eta_{S, \mathrm{f}}, \eta_{T, \mathrm{f}}$ are bounded ('under-fault') measurement noises. We will assume that only one sensor can fail at the time.

Remark 4: The fault model (34) can represent several types of sensor faults of practical interest. For example, $\alpha_{j}=1$ represents 'total outage' of the corresponding sensor and $\alpha_{j} \in(0,1)$ represents 'reduced effectiveness' of the sensor. In addition, sensor 'bias' can be handled by expressing $\eta_{j, \mathrm{f}}=\eta_{j \text {,offset }}+\tilde{\eta}_{j, \mathrm{f}}$, where $\eta_{j \text {,offset }}$ is a constant bias and $\tilde{\eta}_{j, \mathrm{f}}$ has zero mean. For simplicity of exposition, in the remainder of the paper we will assume no bias-type fault, that is, we will assume that the measurement errors $\eta_{j, \mathrm{f}}$ in (34) have zero mean.

In the next two subsections, we will analyse the behaviour of the variables relevant to the switching scheme under the 'working hypothesis' that, in the presence of a faulty sensor, only measurements from healthy sensors are selected by the switching controller. The analysis will finally allow us to derive conditions, in Subsection 7.3, that guarantee that the working hypothesis is satisfied, thus establishing the fault tolerance properties of the scheme.

\subsection{Induction motor variables}

Under the working hypothesis that only measurements from healthy sensors are used by the switching controller, the performance in permanent regime of the induction motor is not affected by a fault in a current sensor. Thus, all equations derived in Section 6.1 (see also Appendix 1) are still valid.

\subsection{Observer variables}

Consider a fault modelled by (34) in the sensor that measures the phase current $i_{R}$. Note from (14), (17) and (18) that only observers 1 and 2 will be affected by this fault whereas observer 3 will remain unaffected. Similarly, a fault in the sensor that measures the phase current $i_{S}$ affects only observers 1 and 3 , and a fault in the sensor that measures the phase current $i_{T}$ affects only observers 2 and 3.

Substituting (34) in the equations of the observers affected by each fault we have, after some calculations (see Appendix $2)$, that the estimation errors in permanent regime $\left(\omega=\omega_{\text {ref }}\right)$ change their dynamics from (29) to

$$
\dot{\tilde{x}}_{l}^{F}=A_{G} \tilde{x}_{l}^{F}+G_{K}\left(\omega_{\text {ref }}\right)\left[E_{l}^{F} \alpha_{F} i_{F}+\eta_{l}^{F}\right], \quad l \in \mathcal{L}^{F}
$$

where $\quad \tilde{x}_{l}^{F}=\left[\begin{array}{llll}\tilde{i}_{\mathrm{s} a, l}^{F} & \tilde{i}_{\mathrm{s} b, l}^{F} & \tilde{\psi}_{\mathrm{r} a, l}^{F} & \tilde{\psi}_{\mathrm{r} b, l}^{F}\end{array}\right]^{\mathrm{T}}$ denotes the 'underfault' estimation errors, $A_{G} \triangleq A\left(\omega_{\text {ref }}\right)+G_{K}\left(\omega_{\text {ref }}\right) C$, $F \in\{R, S, T\}$ denotes the type of fault, and $\mathcal{L}^{F}$ denotes the set of indices of the observers affected by the fault $F$. The set $\mathcal{L}^{F}$, the vectors $E_{l}^{F}$, for $l \in \mathcal{L}^{F}$, and the input $i_{F}$ 
change according to the type of fault as described in the table:

\begin{tabular}{|l|c|c|c|}
\hline $\begin{array}{l}\text { Fault } \\
\text { type }\end{array}$ & $\mathcal{L}^{F}$ & $E_{1}^{F}, I \in \mathcal{L}^{F}$ & $i_{F}$ \\
\hline$R$ & $\{1,2\}$ & $E_{1}^{R}=-\left[\begin{array}{c}1 \\
\sqrt{3} / 3\end{array}\right], E_{2}^{R}=\left[\begin{array}{c}-1 \\
\sqrt{3} / 3\end{array}\right]$ & $i_{R}$ \\
\hline$S$ & $\{1,3\}$ & $E_{1}^{S}=\left[\begin{array}{c}0 \\
-2 / \sqrt{3}\end{array}\right], E_{3}^{S}=\left[\begin{array}{c}1 \\
-\sqrt{3} / 3\end{array}\right]$ & $i_{S}$ \\
\hline$T$ & $\{2,3\}$ & $E_{2}^{T}=\left[\begin{array}{c}0 \\
2 / \sqrt{3}\end{array}\right], E_{3}^{T}=\left[\begin{array}{c}1 \\
\sqrt{3} / 3\end{array}\right]$ & $i_{T}$ \\
\hline
\end{tabular}

The 'under-fault' noise input $\eta_{l}^{F}$ in (35) is a combination of measurement noises; for example, for a fault in the sensor that measures the phase current $i_{R}$, these inputs take the form $\eta_{1}^{R}=\left[\eta_{R, \mathrm{f}}(\sqrt{3} / 3)\left(\eta_{R, \mathrm{f}}+2 \eta_{S, \mathrm{~h}}\right)\right]^{\mathrm{T}}, \quad \eta_{2}^{R}=\left[\eta_{R, \mathrm{f}}-(\sqrt{3} / 3)\right.$ $\left.\left(\eta_{R, \mathrm{f}}+2 \eta_{T, \mathrm{~h}}\right)\right]^{\mathrm{T}}$. Note that (35) is a stable linear system driven by two bounded external inputs: the phase current $i_{F}$ and the 'under-fault' noise $\eta_{l}^{\mathrm{F}}$. Taking Laplace transforms in (35) we have that the components of the flux estimation errors in response to $i_{F}$ satisfy

$$
\left[\begin{array}{c}
\tilde{\psi}_{\mathrm{ra}, l}^{F} \\
\tilde{\psi}_{\mathrm{r} b, l}^{F}
\end{array}\right]_{i_{F}}=\left[\begin{array}{c}
\tilde{H}_{a, l}^{F}(s) \\
\tilde{H}_{b, l}^{F}(s)
\end{array}\right] i_{F}, \quad l \in \mathcal{L}^{F}
$$

where

$$
\begin{aligned}
& \tilde{H}_{a, l}^{F}(s)=\left[\begin{array}{llll}
0 & 0 & 1 & 0
\end{array}\right]\left(s I-A_{G}\right)^{-1} G_{K}\left(\omega_{\mathrm{ref}}\right) E_{l}^{F} \alpha_{F} \\
& \tilde{H}_{b, l}^{F}(s)=\left[\begin{array}{llll}
0 & 0 & 0 & 1
\end{array}\right]\left(s I-A_{G}\right)^{-1} G_{K}\left(\omega_{\mathrm{ref}}\right) E_{l}^{F} \alpha_{F}
\end{aligned}
$$

Since, in permanent regime, $i_{F}$ is a sine wave given by either (74), (75) or (76) in Appendix 1, then the flux estimation error components in (36) will also be sine waves of the form

$$
\begin{aligned}
& {\left[\begin{array}{c}
\tilde{\psi}_{\mathrm{ra}, l}^{F} \\
\tilde{\psi}_{\mathrm{r} b, l}^{F}
\end{array}\right]_{i_{F}}=\left[\begin{array}{cc}
\tilde{a}_{l}^{F} & \sin \left(\omega_{\rho} t+\tilde{\rho}_{a, l}^{F}\right) \\
\tilde{b}_{l}^{F} & \cos \left(\omega_{\rho} t+\tilde{\rho}_{b, l}^{F}\right)
\end{array}\right], \quad l \in \mathcal{L}^{F}} \\
& \tilde{a}_{l}^{F}=\left|\tilde{H}_{a, l}^{F}\left(\mathrm{j} \omega_{\rho}\right)\right| I_{a b}, \quad \tilde{\rho}_{a, l}^{F}=\arg \tilde{H}_{a, l}^{F}\left(\mathrm{j} \omega_{\rho}\right)+\rho_{F} \\
& \tilde{b}_{l}^{F}=\left|\tilde{H}_{b, l}^{F}\left(j \omega_{\rho}\right)\right| I_{a b}, \quad \tilde{\rho}_{b, l}^{F}=\arg \tilde{H}_{b, l}^{F}\left(\mathrm{j} \omega_{\rho}\right)+\rho_{F}-\pi / 2
\end{aligned}
$$

where $\rho_{F}$ is equal to $\rho_{R}, \rho_{S}$ or $\rho_{T}$ (see (74)-(76) in Appendix 1 ) according to the type of fault. Applying the principle of superposition to the system (35), assuming zero initial conditions, yields the following form for the flux estimation errors

$$
\begin{aligned}
& \tilde{\psi}_{\mathrm{r} a, l}^{F}=\tilde{a}_{l}^{F} \sin \left(\omega_{\rho} t+\tilde{\rho}_{a, l}^{F}\right)+\tilde{\eta}_{a, l}^{F} \\
& \tilde{\psi}_{\mathrm{r} b, l}^{F}=\tilde{b}_{l}^{F} \cos \left(\omega_{\rho} t+\tilde{\rho}_{b, l}^{F}\right)+\tilde{\eta}_{b, l}^{F}
\end{aligned}
$$

where we have combined (37) with the components of the flux estimation errors in response to the noise $\eta_{l}^{F}$, which we denote $\tilde{\eta}_{a, l}^{F}$ and $\tilde{\eta}_{b, l}^{F}$. These components are assumed to have zero mean (see Remark 4), although modifications to include sensor bias follow mutatis mutandis at the expense of lengthier calculations. As shown in Appendix 3, (40) can be used to derive the following expression for the observer residuals (23)

$$
\begin{gathered}
\pi_{l}^{F}=\left|\sigma_{l}^{F}+\overline{\left(\tilde{\eta}_{a, l}^{F}\right)^{2}}+\overline{\left(\tilde{\eta}_{b, l}^{F}\right)^{2}}\right|, \quad \text { for } \\
F \in\{R, S, T\}, \quad l \in \mathcal{L}^{F}
\end{gathered}
$$

where

$$
\begin{aligned}
\sigma_{l}^{F} \triangleq & \frac{\left(\tilde{a}_{l}^{F}\right)^{2}}{2}+\frac{\left(\tilde{b}_{l}^{F}\right)^{2}}{2}-\psi_{\mathrm{r}, \mathrm{ref}} \tilde{a}_{l}^{F} \sin \left(\tilde{\rho}_{a, l}^{F}-\rho_{0}\right) \\
& +\psi_{\mathrm{r}, \mathrm{ref}} \tilde{b}_{l}^{F} \sin \left(\tilde{\rho}_{b, l}^{F}-\rho_{0}\right)
\end{aligned}
$$

Thus, we conclude from (41) that, for $F \in\{R, S, T\}$ and $l \in \mathcal{L}^{F}$

$$
\pi_{l}^{F} \geq \bar{\pi}_{l}^{F}, \bar{\pi}_{l}^{F} \triangleq \begin{cases}\sigma_{l}^{F} & \text { if } \sigma_{l}^{F} \geq 0 \\ \| \sigma_{l}^{F}\left|-\left(\epsilon_{a, l}^{F}\right)^{2}-\left(\epsilon_{b, l}^{F}\right)^{2}\right| & \text { if } \sigma_{l}^{F}<0\end{cases}
$$

where $\epsilon_{a, l}^{F}$ and $\epsilon_{b, l}^{F}$ are computed as in Lemma 1 but using the 'under-fault' bounds for the noises. That is, using the dynamic equation (35) instead of (29) and only considering the contribution of the term $\eta_{l}^{F}$ (recall that (40) is obtained by superposition). Since the sampling operation preserves bounds, (43) also yields a lower bound for the sampled signals $\pi_{l}^{F}[k]$ [see (25)], that is

$$
\pi_{l}^{F}[k] \geq \bar{\pi}_{l}^{F}, \quad l \in \mathcal{L}^{F}
$$

Note from (38)-(39) and (70), (73) in Appendix 1 that $\sigma_{l}^{F}$ in (42), and hence the bound (43), can be explicitly computed (for specific values of the reference signals and of the load torque $\tau_{\mathrm{L}}$ ).

\subsection{Fault tolerance conditions}

Under healthy operation, the sampled residual signals $\pi_{j}[k]$, defined in (25), on which the switching strategy bases its decision (26) at each sampling time, are upper bounded as in (33). On the other hand, under a current sensor fault, the sampled residual signals $\pi_{l}^{F}[k]$ associated with the two observers affected by the fault are lower bounded as in (44). Thus, the scheme with switching criterion (26) will be fault tolerant under a fault of type $F$ (where $F \in\{R, S, T\}$ 
indicates a fault in the sensor measuring the phase current $i_{R}$, $i_{S}$ or $\left.i_{T}\right)$ if

$$
\bar{\pi}_{l}^{F}>\bar{\pi}_{j}, \quad \text { for } \quad l \in \mathcal{L}^{F} \text { and } \quad j \in\{1,2,3\} \backslash \mathcal{L}^{F}
$$

where $\bar{\pi}_{l}^{F}$ and $\bar{\pi}_{j}$ were defined in (43) and (33), respectively. Note that, if conditions (45) hold, then observer $j$, $j \in\{1,2,3\} \backslash \mathcal{L}^{F}$, will be chosen by the switching criterion (26) over observers $l, l \in \mathcal{L}^{F}$, which are the ones affected by the faulty sensor measurements.

We observe from (29), (30), (31), (33), (36), (37)-(39) and (42)-(44), that healthy and faulty bounds $\bar{\pi}_{j}$ and $\bar{\pi}_{l}^{F}$, respectively, not only depend on the sensor noise characteristics but also on the reference values, $\psi_{\mathrm{r}, \text { ref }}$ and $\omega_{\text {ref }}$, the load torque $\tau_{\mathrm{L}}$ and the observer gain matrix $G_{K}\left(\omega_{\text {ref }}\right)$ [which, in turn, depends on the observer parameter $K$, see (19) and subsequent equations]. Then, to guarantee fault tolerance, condition (45) should be fulfilled for all operating conditions of the induction motor. This verification can be made numerically specifying the operating region of the motor, for example, $\omega_{\text {ref }} \in\left[\omega_{\min }, \omega_{\max }\right], \tau_{\mathrm{L}} \in\left[\tau_{\mathrm{L}, \min }, \tau_{\mathrm{L}, \max }\right]$ and fixed $\psi_{\mathrm{r}, \mathrm{ref}}$, making an appropriate discretisation of this region and computing the healthy and faulty bounds at each point of the discretisation grid for a given value of the observer parameter $K$. If condition (45) is satisfied for all the points of the discretisation, we can anticipate that it will be valid for the whole operating region since the induction motor variables and the bound expressions change slowly enough to assume that condition (45) still holds between two points of the discretisation. In the following subsection, we illustrate this approach by means of a particular example.

\subsection{Example}

We consider an induction motor with the following parameters: $\quad R_{\mathrm{r}}=0.39923 \Omega, \quad R_{\mathrm{s}}=1.165 \Omega$, $J=0.0812 \mathrm{~kg} \mathrm{~m}^{2}, \quad L_{\mathrm{s}}=0.13995 \mathrm{H}, \quad L_{\mathrm{r}}=0.13995 \mathrm{H}$, $M=0.13421 \mathrm{H}$ and $n_{\mathrm{p}}=2$. The desired constant reference value for the rotor flux amplitude is $\psi_{\mathrm{r} \text {,ref }}=0.888 \mathrm{~Wb}$, and the operating region for the rotor speed and torque load is specified as $\omega_{\text {ref }} \in[125,140] \mathrm{rad} / \mathrm{s}$ and $\tau_{\mathrm{L}} \in[15,30] \mathrm{Nm}$. The sensor noises in (11)-(13) are bounded as $\left|\eta_{R, \mathrm{~h}}\right| \leq 0.04 \mathrm{~A},\left|\eta_{S, \mathrm{~h}}\right| \leq 0.04 \mathrm{~A}$ and $\left|\eta_{T, \mathrm{~h}}\right| \leq 0.04 \mathrm{~A}$. The same bound of $0.04 \mathrm{~A}$ is used for the 'under-fault' noises in (34). We consider total loss of the sensors, that is, $\alpha_{j}=1$ in (34).

Fig. 2 shows the results of a verification test of condition (45) for two values of the observer gain parameter: $K=2.1$ (plots on the left) and $K=3.5$ (plots on the right). We have computed the minimum difference between faulty and healthy bounds when a sensor fault of type $F$, where $F \in\{R, S, T\}$, occurs, defined as

$$
\Delta \pi^{F} \triangleq \min _{l, j}\left\{\bar{\pi}_{l}^{F}-\bar{\pi}_{j}: l \in \mathcal{L}^{F} \quad \text { and } \quad j \in\{1,2,3\} \backslash \mathcal{L}^{F}\right\}
$$

The plots correspond, from top to bottom, to $F=R, F=S$ and $F=T$, respectively.

When $\Delta \pi^{F}>0$, condition (45) is satisfied and we plot a small circle. When $\Delta \pi^{F} \leq 0$, condition (45) is not valid and we plot a larger $*$. We see that for $K=3.5$ there are several points where condition (45) is not valid (in the top right subplot) but for $K=2.1$ condition (45) holds

$$
K=2.1
$$
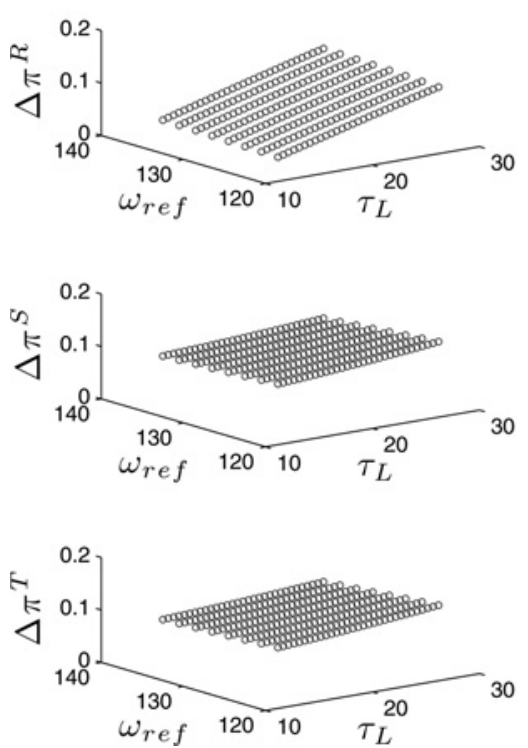
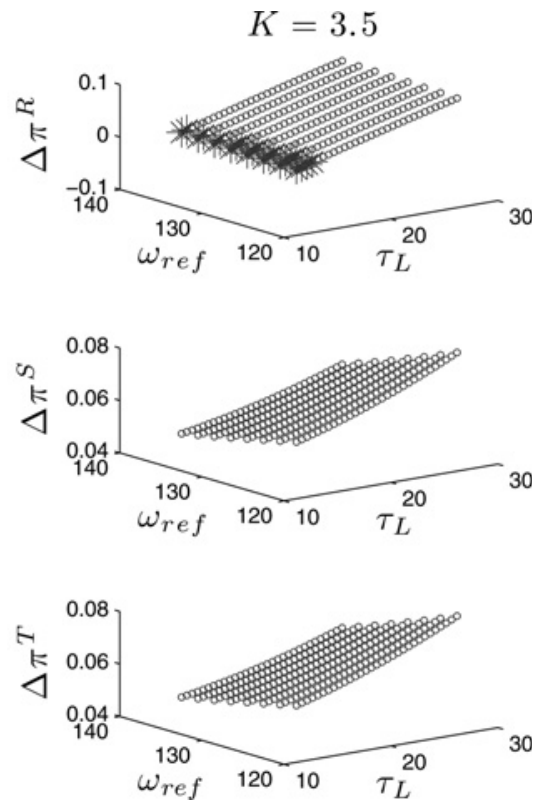

Figure $2 \Delta \pi^{F}$ defined in (46) for $F=R, S, T$ (top to bottom) as a function of $\omega_{\text {ref }}$ and $\tau_{L}$ for two values of the observer parameter $K$ 
for the three phases and all the points of the discretisation. Then, we can anticipate to achieve fault tolerance when the induction motor operates in the predefined region by selecting $K=2.1$ in the observer gain matrix $G_{K}(\omega)$.

We conclude this subsection by suggesting a way to select the filter parameter $T_{H}$ in (24) used to obtain approximately the mean values in the residual signal (23). In faulty conditions, the most significant components of the switching residual signals are those of frequency $2 \omega_{\rho}$, whereas the components of frequency $\omega_{\rho}$ may have more or less prominence depending on the magnitude of the noises [see (85) in Appendix 3]. Hence, the filter should be designed to have sufficient attenuation at the latter frequency. Since $\omega_{\rho}$, computed as in (70) in Appendix 1, is a linear function of the load torque $\tau_{\mathrm{L}}$ and the rotor reference speed $\omega_{\text {ref }}$, a lower bound on $\omega_{\rho}$ for the operating range of Fig. $2\left(\tau_{\mathrm{L}} \in[15,30] \mathrm{Nm}\right.$ and $\omega_{\text {ref }} \in[125$, $140] \mathrm{rad} / \mathrm{s})$ is obtained by setting $\omega_{\text {ref }}=125 \mathrm{rad} / \mathrm{s}$ and (for simplicity) $\tau_{\mathrm{L}}=0 \mathrm{Nm}$ in (70). This yields the bound $\omega_{\rho}>250 \mathrm{rad} / \mathrm{s}$, and hence $1 /\left(\omega_{\rho}\right)<4 \mathrm{~ms}$. For the implementation of the switching strategy we then choose $T_{H}=14 \mathrm{~ms}$ in (24) [several times larger than an upper bound on $1 /\left(\omega_{\rho}\right)$ in the desired operating range], large enough to achieve sufficient attenuation of the harmonic components of the signals but, on the other hand, small enough to avoid the introduction of a large delay in the (implicit) fault detection mechanism.

\section{Application to different vector control strategies}

In this section we describe the FOC and DTFC control techniques, and the corresponding results of the application of the fault-tolerant switching strategy presented in the previous sections.

\subsection{Application to FOC}

We will apply the fault-tolerant switching strategy to the classic FOC technique [6, 7]. The FOC technique first involves the transformation (59) (see Appendix 1) of the vectors $\left(i_{\mathrm{s} a}, i_{\mathrm{s} b}\right)$ and $\left(\psi_{\mathrm{r} a}, \psi_{\mathrm{r} b}\right)$ in the fixed stator frame $(a, b)$ into vectors in a frame $(d, q)$, which rotates with the rotor flux vector $\left(\psi_{\mathrm{r} a}, \psi_{\mathrm{r} b}\right)$. In this new frame the electromagnetic variables of the induction motor satisfy the dynamic equations (61)-(64) in Appendix 1.

The objectives of the FOC methodology are to regulate the rotor flux amplitude $\psi_{\mathrm{r} d}=\sqrt{\psi_{\mathrm{r} a}^{2}+\psi_{\mathrm{r} b}^{2}}$ to a constant reference value $\psi_{\text {r,ref }}$ and to have the rotor speed $\omega$ track a desired reference trajectory $\omega_{\text {ref }}$. These objectives are achieved by combining the following non-linear transformation and linear proportional-integral (PI) control loops

$$
\begin{gathered}
{\left[\begin{array}{l}
u_{d} \\
u_{q}
\end{array}\right]=\sigma L_{\mathrm{s}}\left[\begin{array}{c}
-n_{\mathrm{p}} \omega i_{\mathrm{s} q}-\frac{M}{\tau_{\mathrm{r}}} \frac{i_{\mathrm{s} q}^{2}}{\psi_{\mathrm{r} d}}-\frac{\beta}{\tau_{\mathrm{r}}} \psi_{\mathrm{r} d}+v_{d} \\
n_{\mathrm{p}} \omega i_{\mathrm{s} d}+\frac{M}{\tau_{\mathrm{r}}} \frac{i_{\mathrm{s} q} i_{\mathrm{s} d}}{\psi_{\mathrm{r} d}}+\beta n_{\mathrm{p}} \omega \psi_{\mathrm{r} d}+v_{q}
\end{array}\right]} \\
v_{d}=-k_{d 1}\left(\psi_{\mathrm{r} d}-\psi_{\mathrm{r}, \mathrm{ref}}\right)-k_{d 2} \int_{0}^{t}\left(\psi_{\mathrm{r} d}(s)-\psi_{\mathrm{r}, \mathrm{ref}}\right) \mathrm{d} s \\
v_{q}=-k_{q 1}\left(\tau_{e}-\tau_{\mathrm{ref}}\right)-k_{q 2} \int_{0}^{t}\left(\tau_{e}(s)-\tau_{\mathrm{ref}}(s)\right) \mathrm{d} s \\
\tau_{\mathrm{ref}}=-k_{q 3}\left(\omega-\omega_{\mathrm{ref}}\right)-k_{q 4} \int_{0}^{t}\left(\omega(s)-\omega_{\mathrm{ref}}\right) \mathrm{d} s
\end{gathered}
$$

where $\tau_{e}=\mu \psi_{\mathrm{r} d} i_{\mathrm{s} q}$ and $\omega_{\mathrm{ref}}$ is the desired reference signal for the rotor speed.

Note that the resulting FOC controller is a (dynamic) function of the reference signals and of the states of system (61)-(64) in Appendix 1; moreover, through the transformations (59), it is also a (dynamic) function of the state $x=\left[\begin{array}{llll}i_{\mathrm{s} a} & i_{\mathrm{s} b} & \psi_{\mathrm{r} a} & \psi_{\mathrm{r} b}\end{array}\right]^{\mathrm{T}}$ of system (1). We will denote this function as

$$
u=\mathcal{K}_{\mathrm{FOC}}\left(\omega, x, \psi_{\mathrm{r}, \mathrm{ref}}, \omega_{\mathrm{ref}}\right)
$$

which clearly fits into the generalised form (9). We present below simulation results for the combination of the faulttolerant switching strategy described in the previous sections with the control law (51), implemented using sampled measurements and state estimates as in (27).

\subsection{Simulation results using FOC}

In this section we present simulation results for the complete control system of Fig. 1 where the chosen vector control strategy is the FOC method described in the previous section. The parameters of the induction motor, sensors and observers are as in the example of Section 7.4. The sampling period in (25) is chosen as $T_{\mathrm{s}}=0.07 \mathrm{~ms}$.

The parameters for the PI controllers of the FOC strategy were designed to achieve a given response time, $T_{\psi}, T_{w}$ and $T_{\tau}$, of the respective inner loops, and to reject load perturbation. For the flux magnitude loop we set $T_{\psi}=0.005 \mathrm{~s}$ and the resulting PI parameters in (48) are: $k_{d 1}=522.39$ and $k_{d 2}=1490.2$. For the rotor speed loop we set $T_{w}=0.01 \mathrm{~s}$, which leads to constants $k_{q 3}=9.4081$ and $k_{q 4}=470.76$ in (50), and for the torque inner loop $T_{\tau}=0.005$ s gives $k_{q 1}=2.9657$ and $k_{q 2}=449.78$ in (49).

To simulate a realistic implementation of the controller, the control signals (phase voltages) given by the FOC controller are passed through a three-phase inverter, which gives the phase voltages that feed the induction motor. We 
have selected an inverter that implements a suboscillation modulation method with a carrier frequency of $10 \mathrm{kHz}$ [26].

We consider two simulation scenarios, at 'high' and 'low' rotor speed, respectively.

- For the 'high-speed test', we take the reference signal for rotor speed to be $\omega_{\text {ref }}=130 \mathrm{rad} / \mathrm{s}$. Correspondingly, we select the low-pass filter parameter $T_{H}$ in (24) equal to $T_{H}=14 \mathrm{~ms}$ (see the discussion at the end of Section 7.4). The simulation scenario is as follows. At $t=3 \mathrm{~s}$ a load with $\tau_{\mathrm{L}}=15 \mathrm{Nm}$ is applied. At $t=4 \mathrm{~s}$ an outage of the sensor that measures the phase current $i_{R}$ occurs, that is, its measurement equation changes from (11) to (34) with $\alpha_{R}=1$. Note that both the reference signal values and the load torque are within the ranges considered in Section 7.4; thus, we can conclude from the analysis in that section that the fault-tolerant conditions hold for this scenario. In addition, we further test a value of load torque outside the range considered in Section 7.4 by decreasing the load torque from $\tau_{\mathrm{L}}=15 \mathrm{Nm}$ to $\tau_{\mathrm{L}}=8 \mathrm{Nm}$ at $t=6 \mathrm{~s}$.

The top subplot on the left of Fig. 3 shows the switching signal $\ell_{k}$ resulting from the switching mechanism decision (26). Notice that after the fault at $t=4 \mathrm{~s}$ the switching mechanism only selects observer 3 , as guaranteed by the fault tolerance conditions. The bottom three subplots on the left of Fig. 3 show the sampled residual signals $\pi_{j}[k]$, $j=1,2,3$, defined in (25), corresponding to observers 1,2 and 3, respectively. As analysed in Section 7.2, after the fault at $t=4 \mathrm{~s}$ the residual signals for observers 1 and 2 move quickly to values noticeably away from zero, whereas the residual signal for observer 3 maintains the same small values (near zero) as before the occurrence of the fault. The subplots on the right of Fig. 3 show the response of the rotor speed $\omega$ (top), the applied load torque $\tau_{\mathrm{L}}$ (middle) and the rotor flux magnitude $\psi_{\mathrm{r} d}$ (bottom) under the fault-
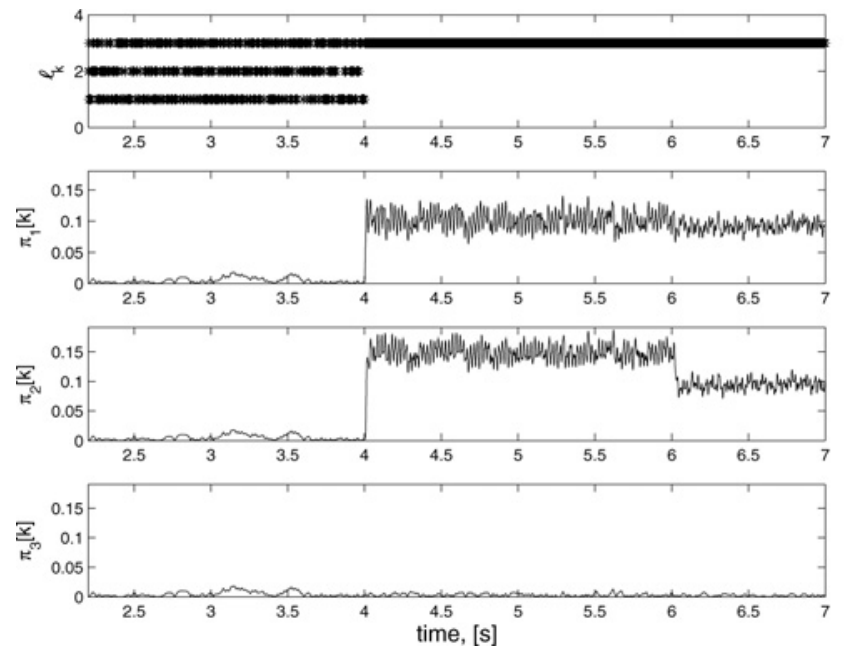

Figure 3 Results of the fault-tolerant FOC control scheme for $\omega_{\text {ref }}=130 \mathrm{rad} / \mathrm{s}$

Left: switching sequence and observer residual signals Right: rotor speed, load torque and rotor flux magnitude tolerant control scheme. Note that the simulated fault at $t=4 \mathrm{~s}$ has no noticeable impact on the rotor speed and flux responses.

- For the 'low-speed test', we take $\omega_{\text {ref }}=30 \mathrm{rad} / \mathrm{s}$. Correspondingly, we select a larger value $T_{H}=50 \mathrm{~ms}$ for the low-pass filter parameter in (24) (see the discussion at the end of Section 7.4). The simulation scenario is as follows. At $t=3 \mathrm{~s}$ a load with $\tau_{\mathrm{L}}=15 \mathrm{~N} \mathrm{~m}$ is applied. At $t=4 \mathrm{~s}$ an outage of the sensor that measures the phase current $i_{R}$ occurs, that is, its measurement equation changes from (11) to (34) with $\alpha_{R}=1$. At $t=6 \mathrm{~s}$ the load torque increases to $\tau_{\mathrm{L}}=22 \mathrm{~N} \mathrm{~m}$. Proceeding as in Section 7.4, it is possible to confirm that the fault tolerance conditions hold for $\omega_{\text {ref }}=30 \mathrm{rad} / \mathrm{s}$ and a load range $\tau_{\mathrm{L}} \in[15,30] \mathrm{Nm}$. The top subplot on the left of Fig. 4 shows the switching signal $\ell_{k}$ resulting from the switching mechanism decision (26). The bottom three subplots on the left of Fig. 4 show the sampled residual signals $\pi_{j}[k], j=1,2,3$, defined in (25), corresponding to observers 1, 2 and 3, respectively. As analysed in Section 7.2, after the fault at $t=4 \mathrm{~s}$ the residual signals for observers 1 and 2 move to values noticeably away from zero, whereas the residual signal for observer 3 maintains the same small values (near zero) as before the occurrence of the fault. The subplots on the right of Fig. 4 show the response of the rotor speed $\omega$ (top), the applied load torque $\tau_{\mathrm{L}}$ (middle) and the rotor flux magnitude $\psi_{\mathrm{r} d}$ (bottom). Note that the simulated fault at $t=4 \mathrm{~s}$ has no noticeable impact on the rotor speed and flux responses.

Finally, Fig. 5 shows the residual variables $\pi_{j}[k]$ for all three observers together with the 'healthy' bound $\bar{\pi}_{1}$ of the form (33) (solid line indicated as (a) in the figure) and the 'under-fault' bounds $\bar{\pi}_{l}^{F}$, for $l=2,3$ of the form (43) (dashed lines indicated as (b) and (c) in the figure). The plot on the left (respectively, right) corresponds to the test scenario for $\omega_{\text {ref }}=130 \mathrm{rad} / \mathrm{s}$ (respectively, $\omega_{\text {ref }}=30 \mathrm{rad} / \mathrm{s}$ ),
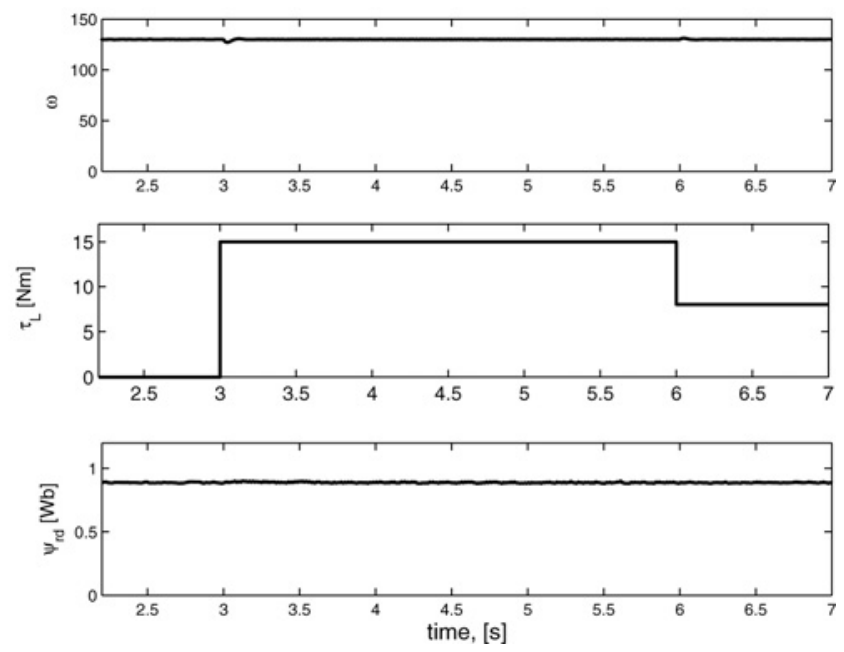

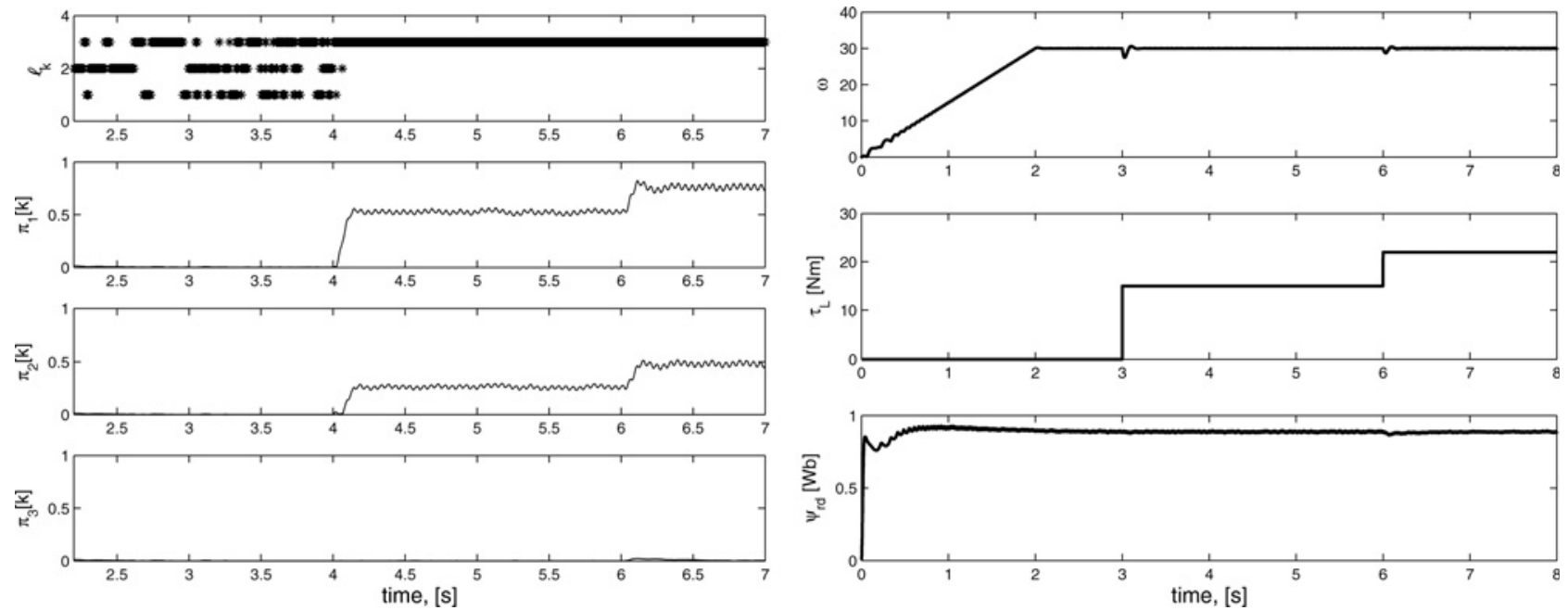

Figure 4 Results of the fault-tolerant FOC control scheme for $\omega_{\mathrm{ref}}=30 \mathrm{rad} / \mathrm{s}$

Left: switching sequence and observer residual signals

Right: rotor speed, load torque and rotor flux magnitude
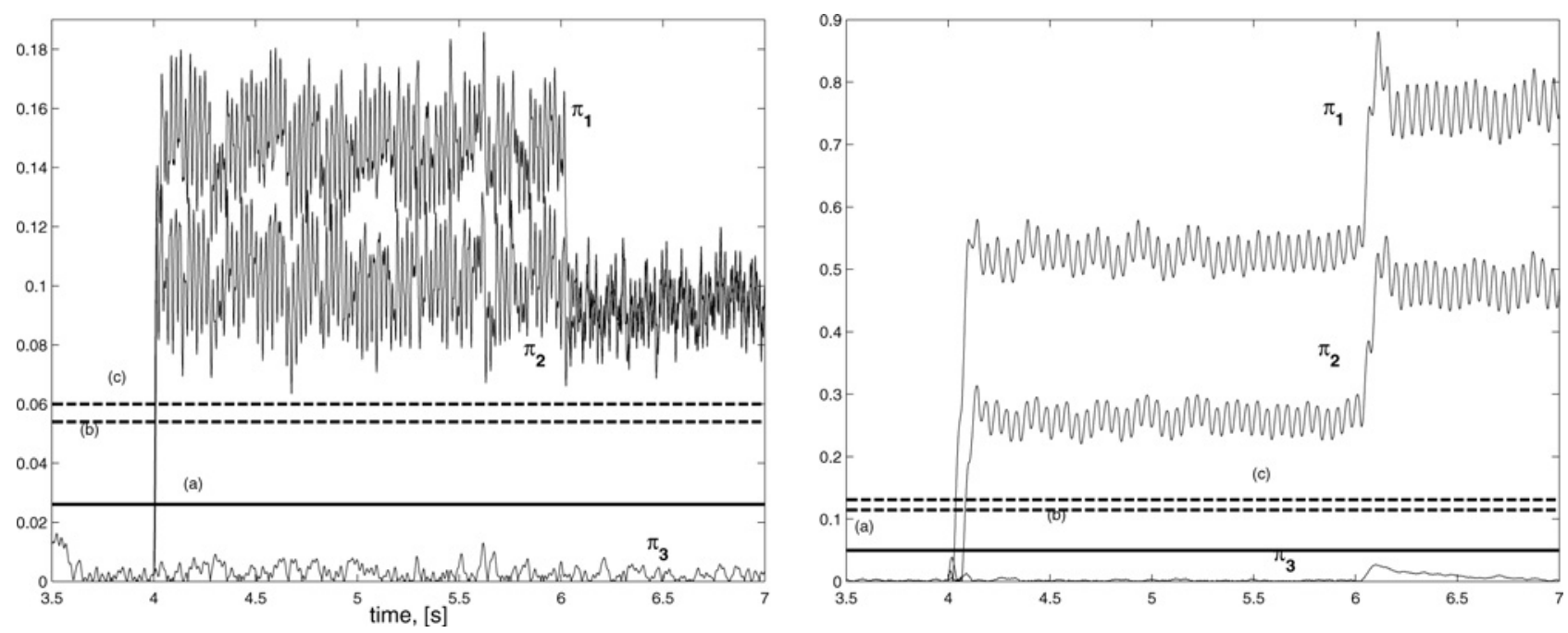

Figure 5 Residual variables and bounds

Left: $\omega_{\text {ref }}=130 \mathrm{rad} / \mathrm{s}$

Right: $\omega_{\text {ref }}=30 \mathrm{rad} / \mathrm{s}$

and all bounds were computed for $\tau_{\mathrm{L}}=15 \mathrm{~N} \mathrm{~m}$. We can see from these plots that the fault tolerance bounds computed in (33) and (43), and required to satisfy (45), are fairly accurate despite the presence of the inverter induced ripple.

Although not shown in this example, a similar situation in terms of the fault tolerance conditions (45) for faults in phases $S$ and $T$ holds true. Thus, the scheme correctly selects the appropriate observer in the event of a fault in any of the phase current sensors provided only one sensor fails at a time.

\subsection{Application to DTFC}

In this section we present the implementation of the faulttolerant switching mechanism embedded in a DTFC

control loop. For this methodology we consider the model of the induction motor in a reference frame fixed with the stator, with components denoted by $(a, b)$, described in Section 2. In the present case, however, we consider the stator, rather than rotor, flux components. That is, we will employ the model

$$
\begin{aligned}
\frac{\mathrm{d} x_{\mathrm{s}}}{\mathrm{d} t} & =A_{\mathrm{s}}(\omega) x_{\mathrm{s}}+B_{\mathrm{s}} u \\
x_{\mathrm{s}} & =\left[\begin{array}{llll}
i_{\mathrm{s} a} & i_{\mathrm{s} b} & \psi_{\mathrm{s} a} & \psi_{\mathrm{s} b}
\end{array}\right]^{\mathrm{T}} \\
u & =\left[\begin{array}{ll}
u_{\mathrm{s} a} & u_{\mathrm{s} b}
\end{array}\right]^{\mathrm{T}}
\end{aligned}
$$

where the state $x_{\mathrm{s}}$ is composed by the stator current and stator flux components, and the input $u$ consists of the 
stator voltage components. The matrices $A_{\mathrm{s}}(\omega)$ and $B_{\mathrm{s}}$ in (52) have the form

$$
\begin{aligned}
A_{\mathrm{s}}(\omega) & =\left[\begin{array}{cc}
-\beta \mathcal{I}+n_{\mathrm{p}} \omega \mathcal{J} & \frac{R_{\mathrm{r}}}{\sigma L_{\mathrm{s}} L_{\mathrm{r}}} \mathcal{I}-\frac{n_{\mathrm{p}} \omega}{\sigma L_{\mathrm{s}}} \mathcal{J} \\
-R_{\mathrm{s}} \mathcal{I} & 0
\end{array}\right], \\
B_{\mathrm{s}} & =\left[\begin{array}{c}
\frac{1}{\sigma L_{\mathrm{s}}} \mathcal{I} \\
\mathcal{I}
\end{array}\right]
\end{aligned}
$$

where $\mathcal{I}$ and $\mathcal{J}$ are as in (5), $\beta=R_{\mathrm{r}} /\left(\sigma L_{\mathrm{r}}\right)+R_{\mathrm{s}} /\left(\sigma L_{\mathrm{s}}\right)$, and the remaining parameters are as defined in Section 2. In addition, the rotor speed $\omega$ satisfies $\mathrm{d} \omega / \mathrm{d} t=$ $(1 / J)\left(\tau_{e}-\tau_{\mathrm{L}}\right)$, where

$$
\tau_{e} \triangleq \frac{3}{2} n_{\mathrm{p}}\left(i_{\mathrm{s} b} \psi_{\mathrm{s} a}-i_{\mathrm{s} a} \psi_{\mathrm{s} b}\right)
$$

is the electromagnetic torque, $J$ is the moment of inertia of the rotor and $\tau_{\mathrm{L}}$ is the load torque.

DTFC is based on the use of hysteresis loops of both electromagnetic torque $\tau_{e}$ in (54) and stator flux magnitude

$$
\psi_{\mathrm{s} d} \triangleq \sqrt{\psi_{\mathrm{s} a}^{2}+\psi_{\mathrm{s} b}^{2}}
$$

to control the output voltage of the inverter through a lookup table. The difference between (estimates of) $\tau_{e}$ and $\psi_{\text {sd }}$ and their corresponding reference signals are passed through separate hysteresis-based comparators to determine logic levels representing the desired action on torque and flux according to the current operating condition. The lookup table then heuristically selects the best output voltage vector according to the position of the stator flux vector $\left(\psi_{\mathrm{s} a}, \psi_{\mathrm{s} b}\right)$ in the $(a, b)$-plane and the desired action on torque and stator flux magnitude [8, 9]. Different lookup tables have been proposed in the literature [27]. Here we consider the well-known scheme of [8].

The reference signal for flux magnitude, denoted as $\psi_{\mathrm{s}, \mathrm{ref}}$, is an external constant signal whereas the reference for electromagnetic torque, $\tau_{\text {ref }}$, although it can be provided externally, is here obtained as an internal signal provided by the PI controller

$$
\tau_{\text {ref }}=P_{w}\left(\omega_{\text {ref }}-\omega\right)+I_{w} \int_{0}^{t}\left(\omega_{\text {ref }}-\omega\right) \mathrm{d} v
$$

where $P_{w}$ and $I_{w}$ are design parameters and $\omega_{\text {ref }}$ is a constant speed reference signal.

The overall DTFC-based controller is thus a function of the rotor speed, the external reference signals $\omega_{\text {ref }}$ and $\psi_{\mathrm{s}, \mathrm{ref}}$ and the state $x_{\mathrm{s}}=\left[i_{\mathrm{s} a} i_{\mathrm{s} b} \psi_{\mathrm{s} a} \psi_{\mathrm{s} b}\right]^{\mathrm{T}}$ of system (52) [required to compute electromagnetic torque (54), flux magnitude
(55) and flux vector position]. We will denote this function as

$$
u=\mathcal{K}_{\mathrm{DTFC}}\left(\omega, x_{\mathrm{s}}, \omega_{\mathrm{ref}}, \psi_{\mathrm{s}, \mathrm{ref}}\right)
$$

which clearly fits into the generalised form (9) upon noting that the states $x$ in (1) and $x_{s}$ in (52) share the first two components, and that the last two components of $x_{\mathrm{s}}$ are $\psi_{\mathrm{s} a}$ and $\psi_{\mathrm{s} b}$.

As mentioned in Remark 2 and Section 5, the DTFC strategy requires the estimation of the stator flux components. For this purpose we implement a Luenberger observer of the form

$$
\frac{\mathrm{d} \hat{x}_{s}}{\mathrm{~d} t}=A_{\mathrm{s}}\left(\omega_{\mathrm{ref}}\right) \hat{x}_{\mathrm{s}}+B_{\mathrm{s}} u+G_{\mathrm{s}}\left(C \hat{x}_{\mathrm{s}}-C z_{\ell_{k}}\right)
$$

where $\hat{x}_{\mathrm{s}}=\left[\hat{i}_{\mathrm{s} a} \hat{i}_{\mathrm{s} b} \hat{\psi}_{\mathrm{s} a} \hat{\psi}_{\mathrm{s} b}\right]^{\mathrm{T}}$ is the state estimate, $A_{\mathrm{s}}\left(\omega_{\mathrm{ref}}\right), B_{\mathrm{s}}$ and $u$ are as in (52) and $C$ is as in (20), and where $z_{\ell_{k}}$ is the output of the observer selected by the switching mechanism (26) during $k T_{\mathrm{s}} \leq t<(k+1) T_{\mathrm{s}}$. The observer gain matrix $G_{\mathrm{s}}$ in (58) is computed such that the eigenvalues of the matrix $A_{\mathrm{s}}\left(\omega_{\text {ref }}\right)+G_{\mathrm{s}} C$ have negative real part.

In the following section, we combine the fault-tolerant switching strategy described in Section 5 with the control law (57) and the stator flux estimates $\hat{\psi}_{\mathrm{s} a}$ and $\hat{\psi}_{\mathrm{s} b}$ obtained from the observer (58), implemented using sampled measurements and state estimates as in (27).

\subsection{Simulation results using DTFC}

For this simulation, the sampling period is chosen as $T_{\mathrm{s}}=0.07 \mathrm{~ms}$ (recall that the sampling period $T_{\mathrm{s}}$ governs both the rate at which the proposed estimate switching mechanism based on (25)-(26) and the 'sampled and held' control (27) are updated). The parameters for the PI controller (56) are taken as $P_{w}=1.9$ and $I_{w}=18.9$. The reference for stator flux magnitude is $\psi_{\mathrm{s} \text {,ref }}=1 \mathrm{~Wb}$.

As for FOC, we consider two simulation scenarios, at high and low rotor speed, respectively.

- For the high-speed test, we take the reference signal for rotor speed to be $\omega_{\text {ref }}=130 \mathrm{rad} / \mathrm{s}$ and the low-pass filter parameter $T_{H}=14 \mathrm{~ms}$ in (24). The simulation scenario is as follows. At $t=3 \mathrm{~s}$ a load with $\tau_{\mathrm{L}}=15 \mathrm{Nm}$ is applied. At $t=3.5 \mathrm{~s}$ an outage of the sensor that measures the phase current $i_{R}$ occurs, that is, its measurement equation changes from (11) to (34) with $\alpha_{R}=1$. At $t=5$ the load torque is decreased to $\tau_{\mathrm{L}}=8 \mathrm{~N} \mathrm{~m}$.

The top subplot on the left of Fig. 6 shows the switching signal $\ell_{k}$ resulting from the switching mechanism decision (26). The bottom three subplots on the left of Fig. 6 show the sampled residual signals $\pi_{j}[k], j=1,2,3$, defined in (25), corresponding to observers 1,2 and 3 , respectively. Notice that both switching sequence and residuals are 

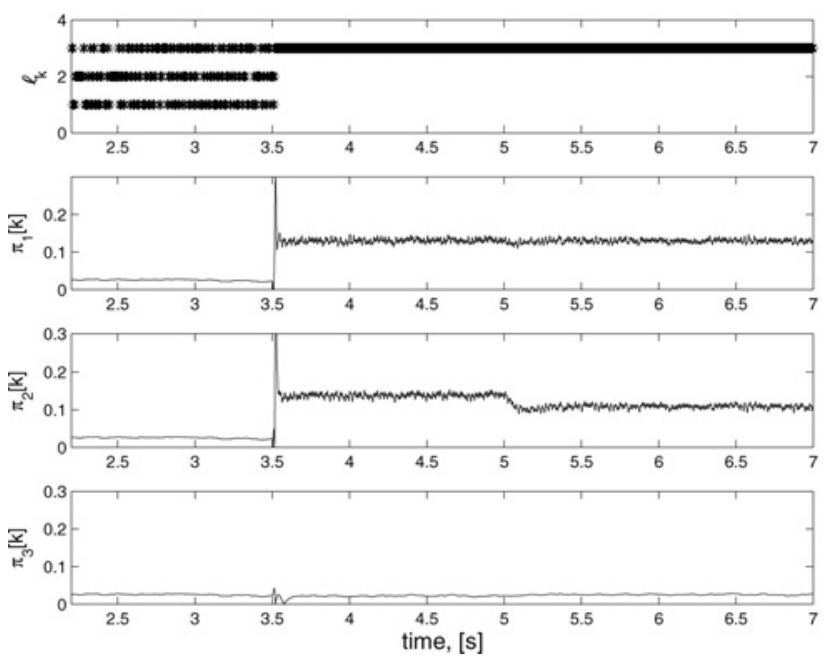

Figure 6 Results of the fault-tolerant DTFC control scheme for $\omega_{\text {ref }}=130 \mathrm{rad} / \mathrm{s}$

Left: switching sequence and observer residual signals

Right: rotor speed, load torque and stator flux magnitude

similar to the analogous ones obtained for FOC in Fig. 3. The subplots on the right of Fig. 6 show the response of the rotor speed $\omega$ (top), the applied load torque $\tau_{\mathrm{L}}$ (middle) and the stator flux magnitude $\psi_{\text {sd }}$ (bottom) under the fault-tolerant control scheme. Note that the simulated fault at $t=3.5 \mathrm{~s}$ has no noticeable impact on the rotor speed and stator flux responses.

- For the low speed test, we take $\omega_{\text {ref }}=30 \mathrm{rad} / \mathrm{s}$ and select the value $T_{H}=50 \mathrm{~ms}$ for the low-pass filter parameter in (24). The simulation scenario is as follows. At $t=3 \mathrm{~s} \mathrm{a}$ load with $\tau_{\mathrm{L}}=15 \mathrm{Nm}$ is applied. At $t=5 \mathrm{~s}$ an outage of the sensor that measures the phase current $i_{R}$ occurs, that is, its measurement equation changes from (11) to (34) with $\alpha_{R}=1$. At $t=6 \mathrm{~s}$ the load torque increases to $\tau_{\mathrm{L}}=22 \mathrm{Nm}$.
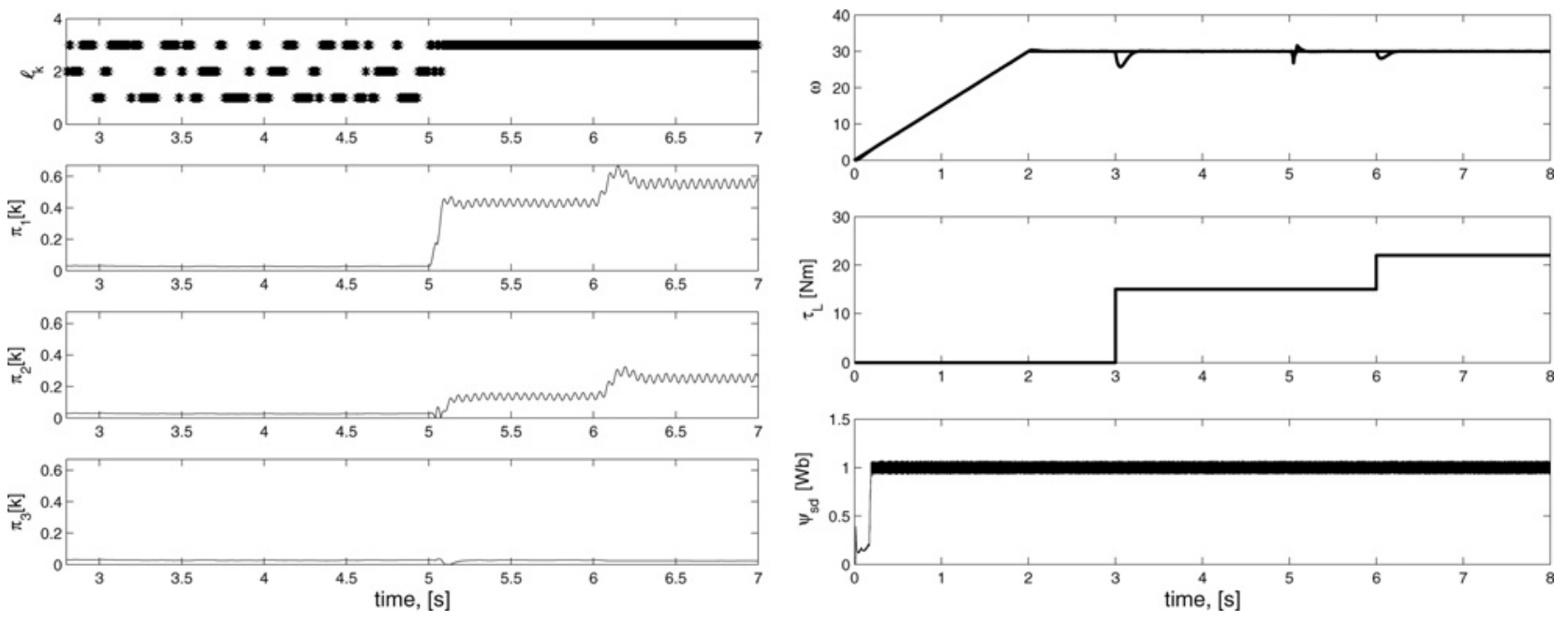

Figure 7 Results of the fault-tolerant DTFC control scheme for $\omega_{\text {ref }}=30 \mathrm{rad} / \mathrm{s}$

Left: switching sequence and observer residual signals

Right: rotor speed, load torque and stator flux magnitude 
to the inverter-induced ripple, as shown in the presented simulation studies, we have performed further tests including parameter variations in the induction motor model. More specifically, the simulation scenarios presented above for both FOC and DTFC at high and low speed have been repeated after increasing by $20 \%$, one by one and all simultaneously, the following parameters: $R_{s}, R_{r}$ and the leakage inductances $L_{\mathrm{s}}-M, L_{\mathrm{r}}-M$. We have also repeated all tests with increased current sensor noise $\left(\left|\eta_{R, \mathrm{~h}}\right| \leq 0.108 \mathrm{~A},\left|\eta_{S, \mathrm{~h}}\right| \leq 0.108 \mathrm{~A}\right.$ and $\left.\left|\eta_{T, \mathrm{~h}}\right| \leq 0.108 \mathrm{~A}\right)$ and with added speed sensor noise of $0.5 \%$ (assuming the use of a relatively precise encoder). The results of those studies of parameter variation, increased current noise and added speed measurement noise (not presented here for space reasons) showed that the scheme continued to perform satisfactorily and preserved the fault tolerance capabilities in all tested cases.

\section{Conclusions}

We have proposed the use of a strategy based on multisensor switching for sensor fault-tolerant vector control of induction motors. The proposed strategy combines stator current sensors, rotor flux observers and a switching mechanism that selects the sensors-observer pair with the smallest error between the estimated flux magnitude and a desired flux reference. The estimates provided by the selected pair are used by a vector controller (such as FOC and DTFC) to implement the control law. We have provided precheckable conditions that guarantee fault tolerance under an abrupt fault of one of the current sensors. These conditions are such that the observers that use measurements from the faulty sensor are automatically avoided by the switching mechanism, thus maintaining good performance levels even in the presence of a faulty sensor. The results have been illustrated by simulation examples of the application of the fault-tolerant switching scheme to FOC and DTFC control. Future work will consider the combination of the present scheme with speed-sensorless strategies employing speed estimators in order to achieve fault tolerance against speed sensor faults.

\section{References}

[1] LUO R., YIH C.-C., SU K.: 'Multisensor fusion and integration: approaches, applications, and future research directions', IEEE Sensors J., 2002, 2, (2), pp. 107-119

[2] MARtínez J., zhUO X., DE dONÁ J., SERON M.: 'Multisensor switching strategy for automotive longitudinal control'. Proc. 2006 American Control Conf., Minneapolis, MN, USA, 2006

[3] SERON M., ZHUO X., DE DONÁ J., MARTínez J.: 'Multisensor switching control strategy with fault tolerance guarantees', Automatica, 2008, 44, (1), pp. 88-97
[4] LEONHARD W.: 'Control of electrical drives' (Springer, Berlin, Germany, 1996)

[5] LEE K.-S., RYU J.-S.: 'Instrument fault detection and compensation scheme for direct torque controlled induction motor drives', IEE Proc. Control Theory Appl., 2003, 150, (4), pp. 376-382

[6] BLASCHKE F.: 'The principle of field orientation applied to the new transvector closed-loop control system for rotating field machines', Siemens-Rev., 1972, 39, pp. 217-220

[7] MARINO R., PERESADA S., VALIGI P.: 'Adaptive input-output linearizing control of induction motors', IEEE Trans. Autom. Control, 1993, 38, (2), pp. 208-221

[8] TAKAHASHI I., NOGUCHI T.: 'A new quick response and highefficiency control strategy of an induction motor', IEEE Trans. Ind. Appl., 1986, 22, pp. 820-827

[9] DEPENBROCK M.: 'Direct self-control (DSC) of inverter-fed induction machine', IEEE Trans. Power Electron., 1988, 3, pp. $420-429$

[10] KUBOTA H., MATSUSE K.: 'Speed sensorless field-oriented control of induction motor with rotor resistance adaptation', IEEE Trans. Ind. Appl., 1994, 30, (5), pp. 1219-1224

[11] BeNNETT S.M., PATTON R.J., DALEY S., NEWTON D.A.: 'Torque and flux estimation for a rail traction system in the presence of intermittent sensor faults'. UKACC Int. Conf. on Control '96, September 1996, vol. 1, pp. 72-77

[12] SEPE JR. R., FAHIMI B., MORRISON C., MILLER J.: 'Fault tolerant operation of induction motor drives with automatic controller reconfiguration'. IEEE Int. Electric Machines and Drives Conf., IEMDC 2001, 2001, pp. 156-162

[13] TAHAMI F., SHOJAEI A., KHATIR D.A.: 'A diversity based reconfigurable method for fault tolerant control of induction motors'. Proc. Int. Symp. on Power Electronics, Electrical Drives, Automation and Motion, 2006, pp. 12-17

[14] DIALLO D., BENBOUZID M.E.H., MAKOUF A.: 'A fault-tolerant control architecture for induction motor drives in automotive applications', IEEE Trans. Veh. Technol., 2004, 53, (6), pp. 1847-1855

[15] BeNBOUZID M.E.H., DIALLO D., ZERAOULIA M.: 'Advanced faulttolerant control of induction-motor drives for ev/hev traction applications: from conventional to modern and intelligent control techniques', IEEE Trans. Veh. Technol., 2007, 56, (2), pp. 519-528

[16] SERON M., ROMERO M., DE DONÁ J.: 'Sensor fault tolerant control of induction motors'. Proc. 17th IFAC World Congress, Seoul, July 2008 
[17] ROMERO M., SERON M., DE DONÁ J.: 'Sensor fault tolerant direct torque and flux control of induction motors'. Seventh IFAC Symp. on Fault Detection, Supervision and Safety of Technical Processes, SAFEPROCESS'09, Barcelona, Spain, 30 June-3 July 2009

[18] ROMERO M., SERON M.: 'Speed-sensorless vector control of induction motors with fault tolerance against current sensor failure'. XIII Workshop on Information Processing and Control, RPIC'09, Rosario, Argentina, 16-18 September 2009

[19] KRAUSE P., WASYNCZUK O., SUDHOFF S.: 'Analysis of electric machinery' (IEEE press, New York, 1995)

[20] ortega R., barabanov N., escobar valderrama G.: 'Direct torque control of induction motors: stability analysis and performance improvement', IEEE Trans. Autom. Control, 2001, 46, (8), pp. 1209-1222

[21] PAPAFOtIOU G., GEYeR T., MORARI M.: 'Optimal direct torque control of three-phase symmetric induction motors'. Proc. IEEE Conf. on Decision and Control, 2004, vol. 2, pp. $1860-1865$

[22] ROMERO M., BRASLAVSKY J., VALLA M.: 'A ripple minimization strategy for direct torque and flux control of induction motors using sliding modes'. Proc. 15th IFAC World Congress on Automatic Control, Barcelona, Spain, July 2002

[23] YAN Z., JIN C., UTKIN V.: 'Sensorless sliding-mode control of induction motors', IEEE Trans. Ind. Electron., 2000, 47, pp. 1287-1297

[24] VERGHESE G., SANDERS S.: 'Observers for flux estimation in induction machines', IEEE Trans. Ind. Electron., 1988, 35, (1), pp. 85-94

[25] KOFMAN E., HAIMOVICH H., SERON M.: 'A systematic method to obtain ultimate bounds for perturbed systems', Int. J. Control, 2007, 80, (2), pp. 167-178

[26] FELICIONI F., PÉREZ T., MOLINA H., JUNCO S.: 'Simudrives: a tool for computer-aided simulation of electrical drives and motion control systems'. XVIII Congr. Argentino Control Automático, Argentina, 2002, available from www.eie. fceia.unr.edu.ar/ curmot/sis_curmot.html

[27] BUJA G., CASADEI D., SERRA G.: 'Direct stator flux and torque control of an induction motor: theoretical analysis and experimental results'. Proc. 24th Annual Conf. IEEE Ind. Electronics Society, Aachen, Germany, 1998, vol. 1, pp. T50-T64

[28] BeRTOLUZZO M., BUJA G., MENIS R.: 'Analytical formulation of the direct control of induction motor drives'. IEEE Int. Symp. Industrial Electronics, Bled, Slovenia, 1999, pp. Ps14-Ps20

\section{Appendix 1: permanent regime values}

To obtain the permanent regime values, it will be convenient to transform the induction motor variables from the fixed stator frame $(a, b)$ into a frame $(d, q)$ oriented with the rotor flux vector $\psi_{\mathrm{r}}$ (i.e. the frame rotates with the rotor flux vector and the component $\left.\psi_{\mathrm{r}, q}=0\right)$. Defining $\rho=\arctan \left(\psi_{\mathrm{r} b} / \psi_{\mathrm{r} a}\right)$, the transformation is

$$
\left[\begin{array}{l}
y_{d} \\
y_{q}
\end{array}\right]=\left[\begin{array}{cc}
\cos \rho & \sin \rho \\
-\sin \rho & \cos \rho
\end{array}\right]\left[\begin{array}{l}
y_{a} \\
y_{b}
\end{array}\right]
$$

where $y$ refers to any of the variables of interest $\left(i_{\mathrm{s}}, \psi_{\mathrm{s}}, \psi_{\mathrm{r}}\right.$ and $u$ ). Note that, since component $\psi_{\mathrm{r}, q}=0$, then

$$
\psi_{\mathrm{r} d}=\sqrt{\psi_{\mathrm{r} a}^{2}+\psi_{\mathrm{r} b}^{2}}
$$

that is, $\psi_{\mathrm{r} d}$ is the magnitude of the rotor flux. In this reference frame, the motor variables satisfy the following differential equations

$$
\begin{gathered}
\frac{\mathrm{d} \psi_{\mathrm{r} d}}{\mathrm{~d} t}=-\frac{1}{\tau_{\mathrm{r}}} \psi_{\mathrm{r} d}+\frac{M}{\tau_{\mathrm{r}}} i_{\mathrm{s} d} \\
\frac{\mathrm{d} i_{\mathrm{s} d}}{\mathrm{~d} t}=-\gamma i_{\mathrm{s} d}+\frac{\beta}{\tau_{\mathrm{r}}} \psi_{\mathrm{r} d}+n_{\mathrm{p}} \omega i_{\mathrm{s} q}+\frac{M}{\tau_{\mathrm{r}}} \frac{i_{\mathrm{s} q}^{2}}{\psi_{\mathrm{r} d}}+\frac{1}{\sigma L_{\mathrm{s}}} u_{d} \\
\frac{\mathrm{d} i_{\mathrm{s} q}}{\mathrm{~d} t}=-\gamma i_{\mathrm{s} q}-\beta n_{\mathrm{p}} \omega \psi_{\mathrm{r} d}-n_{\mathrm{p}} \omega i_{\mathrm{s} d}-\frac{M}{\tau_{\mathrm{r}}} \frac{i_{\mathrm{s} q} i_{\mathrm{s} d}}{\psi_{\mathrm{r} d}}+\frac{1}{\sigma L_{\mathrm{s}}} u_{q}
\end{gathered}
$$

$$
\begin{aligned}
& \frac{\mathrm{d} \omega}{\mathrm{d} t}=\mu \psi_{\mathrm{r} d} i_{\mathrm{s} q}-\frac{\tau_{\mathrm{L}}}{J} \\
& \frac{\mathrm{d} \rho}{\mathrm{d} t}=n_{\mathrm{p}} \omega+\frac{M}{\tau_{\mathrm{r}}} \frac{i_{\mathrm{s} q}}{\psi_{\mathrm{r} d}}
\end{aligned}
$$

where $\quad \gamma=M^{2} R_{\mathrm{r}} /\left(\sigma L_{\mathrm{s}} L_{\mathrm{r}}^{2}\right)+R_{\mathrm{s}} /\left(\sigma L_{\mathrm{s}}\right), \quad \beta=M /\left(\sigma L_{\mathrm{r}} L_{\mathrm{s}}\right)$ and $\mu=n_{\mathrm{p}} M /\left(J L_{\mathrm{r}}\right)$.

For constant $\tau_{\mathrm{L}}$ and assuming that the motor has achieved a given operational condition defined by constant values of $\omega=\omega_{\text {ref }}$ and $\psi_{\mathrm{r} d}=\psi_{\mathrm{r} \text {,ref }}$, the corresponding constant values for the current components, obtained from (61) and (64), are

$$
\begin{gathered}
i_{\mathrm{s} d}=\frac{\psi_{\mathrm{r}, \mathrm{ref}}}{M} \\
i_{\mathrm{s} q}=\frac{\tau_{\mathrm{L}}}{J \mu \psi_{\mathrm{r}, \mathrm{ref}}}
\end{gathered}
$$

Using the above values, we can compute the reference value $\psi_{\mathrm{s} \text {,ref }}$ for the magnitude of the stator flux vector (to be used in the DTFC strategy) by means of the following 
relations [28]

$$
\psi_{\mathrm{s} d}=\frac{M}{L_{\mathrm{r}}} \psi_{\mathrm{r}, \mathrm{ref}}+\sigma L_{\mathrm{s}} i_{\mathrm{s} d}, \quad \psi_{\mathrm{s} q}=\sigma L_{\mathrm{s}} i_{\mathrm{s} q}
$$

Then, from (68), (66) and (67), we obtain

$$
\psi_{\mathrm{s}, \mathrm{ref}}=\sqrt{\left(\frac{M}{L_{\mathrm{r}}}+\frac{\sigma L_{\mathrm{s}}}{M}\right)^{2} \psi_{\mathrm{r}, \mathrm{ref}}^{2}+\left(\frac{\sigma L_{\mathrm{s}} \tau_{\mathrm{L}}}{J \mu \psi_{\mathrm{r}, \mathrm{ref}}}\right)^{2}}
$$

Once the $(d, q)$ permanent regime values are computed, we retrieve the variables in the fixed frame $(a, b)$ integrating $\rho$ in (65) from $\rho(0)=\rho_{0}$ to obtain

$$
\rho(t)=\omega_{\rho} t+\rho_{0}, \quad \omega_{\rho} \triangleq n_{\mathrm{p}} \omega_{\mathrm{ref}}+\frac{M}{\tau_{\mathrm{r}}} \frac{i_{s q}}{\psi_{\mathrm{r}, \mathrm{ref}}}
$$

where $i_{\text {sq }}$ is given (67). Then, using (59), (60) and (70) yields

$$
\begin{aligned}
& \psi_{\mathrm{r} a}(t)=\psi_{\mathrm{r}, \mathrm{ref}} \cos \left(\omega_{\rho} t+\rho_{0}\right) \\
& \psi_{\mathrm{r} b}(t)=\psi_{\mathrm{r}, \mathrm{ref}} \sin \left(\omega_{\rho} t+\rho_{0}\right)
\end{aligned}
$$

Similarly, using (66), (67), (70) and (59) we obtain, after some trigonometric manipulations

$$
\begin{aligned}
& i_{\mathrm{s} a}(t)=I_{a b} \sin \left(\omega_{\rho} t+\rho_{0}+\rho_{a b}\right) \\
& i_{\mathrm{s} b}(t)=-I_{a b} \cos \left(\omega_{\rho} t+\rho_{0}+\rho_{a b}\right)
\end{aligned}
$$

where

$$
I_{a b}=\sqrt{\frac{\psi_{\mathrm{r}, \mathrm{ref}}^{2}}{M^{2}}+\frac{\tau_{L}^{2}}{J^{2} \mu^{2} \psi_{\mathrm{r}, \mathrm{ref}}^{2}}}, \rho_{a b}=\arccos \frac{-1}{\sqrt{1+\left(J \mu \psi_{\mathrm{ref}}^{2} / M \tau_{\mathrm{L}}\right)^{2}}}
$$

Finally, the phase currents $i_{R}, i_{S}$ and $i_{T}$ can be obtained from (8), (10) and (72) as

$$
\begin{aligned}
& i_{R}(t)=i_{\mathrm{s} a}(t)=I_{a b} \sin \left(\omega_{\rho} t+\rho_{R}\right), \quad \rho_{R}=\rho_{0}+\rho_{a b} \\
& i_{S}(t)=I_{a b} \sin \left(\omega_{\rho} t+\rho_{S}\right), \quad \rho_{S}=\rho_{0}+\rho_{a b}-2 \pi / 3 \\
& i_{T}(t)=I_{a b} \sin \left(\omega_{\rho} t+\rho_{T}\right), \quad \rho_{T}=\rho_{0}+\rho_{a b}-4 \pi / 3
\end{aligned}
$$

\section{Appendix 2: computation of the estimation errors under fault}

We explain in this appendix the derivation of (35) and its subsequent table, associated with the dynamics of the 'under-fault' estimation errors.
Expanding the equations in (17) by substitution of the expressions (14)-(16) yields

$$
\begin{array}{r}
i_{\mathrm{s} a, 1}=i_{R, m}, \quad i_{\mathrm{s} b, 1}=\frac{\sqrt{3}}{3}\left(i_{R, m}+2 i_{S, m}\right) \\
i_{\mathrm{s} a, 2}=i_{R, m}, \quad i_{\mathrm{s} b, 2}=\frac{\sqrt{3}}{3}\left(-i_{R, m}-2 i_{T, m}\right) \\
i_{\mathrm{sa}, 3}=-i_{S, m}-i_{T, m}, \quad i_{\mathrm{s} b, 3}=\frac{\sqrt{3}}{3}\left(i_{S, m}-i_{T, m}\right)
\end{array}
$$

We next substitute the above equations and the 'underfault' measurement equations (34) in the dynamics (18) for each of the three observers and further use the fact that

$$
C \hat{x}_{j}=C\left[\hat{i}_{\mathrm{s} a, j} \hat{i}_{\mathrm{s} b, j} \hat{\psi}_{\mathrm{r} a, j} \hat{\psi}_{\mathrm{r} b, j}\right]^{\mathrm{T}}=\left[\hat{i}_{\mathrm{s} a, j} \hat{i}_{\mathrm{s} b, j}\right]^{\mathrm{T}}
$$

for $j=1,2,3$ and $C$ as defined in (20).

We thus have for observer 1

$$
\begin{aligned}
\dot{\hat{x}}_{1}^{F}= & A(\omega) \hat{x}_{1}^{F}+B u+G_{K}(\omega)[C \hat{x}_{1}^{F}-\underbrace{\left[\begin{array}{c}
i_{R} \\
(\sqrt{3} / 3)\left(i_{R}+2 i_{S}\right)
\end{array}\right]}_{\left[i_{a} i_{b}\right]^{\mathrm{T}}} \\
& -\left[\begin{array}{c}
-\alpha_{R} i_{R} \\
-(\sqrt{3} / 3)\left(\alpha_{R} i_{R}+2 \alpha_{S} i_{S}\right)
\end{array}\right] \\
& +G_{K}(\omega)\left[\begin{array}{c}
-\eta_{R, \mathrm{f}} \\
-(\sqrt{3} / 3)\left(\eta_{R, \mathrm{f}}+2 \eta_{S, \mathrm{f}}\right)
\end{array}\right] \\
= & {\left[A(\omega)+G_{K}(\omega) C\right] \hat{x}_{1}^{F}+B u-G_{K}(\omega) } \\
& \times[\underbrace{\left[\begin{array}{c}
i_{a} \\
i_{b}
\end{array}\right]}_{C x}+\left[\begin{array}{c}
-1 \\
-\sqrt{3} / 3
\end{array}\right] \alpha_{R} i_{R}+\left[\begin{array}{c}
0 \\
-2 / \sqrt{3}
\end{array}\right] \alpha_{S} i_{S}+\text { noises }
\end{aligned}
$$

Hence, the 'under-fault' estimation error $\tilde{x}_{1}^{F}=x-\hat{x}_{1}^{F}$ satisfies, using the induction motor equation 1 and the above equation

$$
\begin{aligned}
\dot{\tilde{x}}_{1}^{F}= & {\left[A(\omega)+G_{K}(\omega) C\right] \tilde{x}_{1}^{F}+G_{K}(\omega) } \\
& \times\left[\left[\begin{array}{c}
-1 \\
-\sqrt{3} / 3
\end{array}\right] \alpha_{R} i_{R}+\left[\begin{array}{c}
0 \\
-2 / \sqrt{3}
\end{array}\right] \alpha_{S} i_{S}+\text { noises }\right]
\end{aligned}
$$

Similarly, the 'under-fault' estimation errors for observers 2 
and 3 satisfy

$$
\begin{aligned}
& \dot{\tilde{x}}_{2}^{F}=\left[A(\omega)+G_{K}(\omega) C\right] \tilde{x}_{2}^{F}+G_{K}(\omega)\left[\left[\begin{array}{c}
-1 \\
\sqrt{3} / 3
\end{array}\right] \alpha_{R} i_{R}\right. \\
& \left.+\left[\begin{array}{c}
0 \\
2 / \sqrt{3}
\end{array}\right] \alpha_{T} i_{T}+\text { noises }\right]
\end{aligned}
$$

$$
\begin{aligned}
& \dot{\tilde{x}}_{3}^{F}=\left[A(\omega)+G_{K}(\omega) C\right] \tilde{x}_{3}^{F}+G_{K}(\omega)\left[\left[\begin{array}{c}
1 \\
-\sqrt{3} / 3
\end{array}\right] \alpha_{S} i_{S}\right. \\
& \left.+\left[\begin{array}{c}
1 \\
\sqrt{3} / 3
\end{array}\right] \alpha_{T} i_{T}+\text { noises }\right]
\end{aligned}
$$

Equation (35) and subsequent table easily follow (81)-(83) evaluated at $\omega=\omega_{\text {ref }}$.

\section{Appendix 3: computation of the residual variables under fault}

We will use (40) to derive an expression for the observer residuals (23) under fault of each of the phase current sensors. The signals (23) can be written [see (32)] as

$$
\pi_{l}^{F}=\overline{\mid\left(\tilde{\psi}_{\mathrm{r} a, l}^{F}\right)^{2}}+\overline{\left(\tilde{\psi}_{\mathrm{r} b, l}^{F}\right)^{2}}-\overline{2 \psi_{\mathrm{r} a} \tilde{\psi}_{\mathrm{r} a, l}^{F}}-\overline{2 \psi_{\mathrm{r} b} \tilde{\psi}_{\mathrm{r} b, l}^{F}} \mid, \quad l \in \mathcal{L}^{F}
$$

where $F \in\{R, S, T\}$, according to the type of fault, and $\psi_{\mathrm{r} a}$, $\psi_{\mathrm{r} b}$ satisfy (71). Substituting (71) and (40) into (84) and using the trigonometric expressions

$$
\begin{gathered}
\sin \alpha \cos \beta=\frac{1}{2}[\sin (\alpha+\beta)+\sin (\alpha-\beta)] \\
\sin ^{2} \alpha=\frac{1}{2}[1-\cos (2 \alpha)]=\frac{1}{2}[1+\sin (2 \alpha-\pi / 2)] \\
\cos ^{2} \alpha=\frac{1}{2}[1+\cos (2 \alpha)]=\frac{1}{2}[1-\sin (2 \alpha-\pi / 2)]
\end{gathered}
$$

the different terms under the 'mean-value bars' on the right- hand side of (84) can be written as

$$
\begin{aligned}
\left(\tilde{\psi}_{\mathrm{r} a, l}^{F}\right)^{2}= & \frac{\left(\tilde{a}_{l}^{F}\right)^{2}}{2}\left[1+\sin \left(2 \omega_{\rho} t+2 \tilde{\rho}_{a, l}^{F}-\pi / 2\right)\right] \\
& +\left(\tilde{\eta}_{a, l}^{F}\right)^{2}+2 \tilde{\eta}_{a, l}^{F} \tilde{a}_{l}^{F} \sin \left(\omega_{\rho} t+\tilde{\rho}_{a, l}^{F}\right) \\
\left(\tilde{\psi}_{\mathrm{r} b, l}^{F}\right)^{2}= & \frac{\left(\tilde{b}_{l}^{F}\right)^{2}}{2}\left[1-\sin \left(2 \omega_{\rho} t+2 \tilde{\rho}_{b, l}^{F}-\pi / 2\right)\right] \\
& +\left(\tilde{\eta}_{b, l}^{F}\right)^{2}+2 \tilde{\eta}_{b, l}^{F} \tilde{b}_{l}^{F} \cos \left(\omega_{\rho} t+\tilde{\rho}_{b, l}^{F}\right) \\
2 \psi_{\mathrm{r} a} \tilde{\psi}_{\mathrm{r} a, l}^{F}= & \psi_{\mathrm{r}, \mathrm{ref}} \tilde{a}_{l}^{F} \sin \left(2 \omega_{\rho} t+\rho_{0}+\tilde{\rho}_{a, l}^{F}\right) \\
& +\psi_{\mathrm{r}, \mathrm{ref}} \tilde{a}_{l}^{F} \sin \left(\tilde{\rho}_{a, l}^{F}-\rho_{0}\right) \\
& +2 \tilde{\eta}_{a, l}^{F} \psi_{\mathrm{r}, \mathrm{ref}} \cos \left(\omega_{\rho} t+\rho_{0}\right) \\
2 \psi_{\mathrm{r} b} \tilde{\psi}_{\mathrm{r} b, l}^{F}= & \psi_{\mathrm{r}, \mathrm{ref}} \tilde{b}_{l}^{F} \sin \left(2 \omega_{\rho} t+\rho_{0}+\tilde{\rho}_{b, l}^{F}\right) \\
& -\psi_{\mathrm{r}, \mathrm{ref}} \tilde{b}_{l}^{F} \sin \left(\tilde{\rho}_{b, l}^{F}-\rho_{0}\right)+2 \tilde{\eta}_{b, l}^{F} \psi_{\mathrm{r}, \mathrm{ref}} \sin \left(\omega_{\rho} t+\rho_{0}\right)
\end{aligned}
$$

with $\rho_{0}$ as defined in (70) in Appendix 1. Taking mean values [recall the discussion after (24)], we obtain

$$
\begin{aligned}
\overline{\left(\tilde{\psi}_{\mathrm{r} a, l}^{F}\right)^{2}} & =\frac{\left(\tilde{a}_{l}^{F}\right)^{2}}{2}+\overline{\left(\tilde{\eta}_{a, l}^{F}\right)^{2}} \\
\overline{\left(\tilde{\psi}_{\mathrm{r} b, l}^{F}\right)^{2}} & =\frac{\left(\tilde{b}_{l}^{F}\right)^{2}}{2}+\overline{\left(\tilde{\eta}_{b, l}^{F}\right)^{2}} \\
\overline{-2 \psi_{\mathrm{r} a} \tilde{\psi}_{\mathrm{r} a, l}^{F}} & =-\psi_{\mathrm{r}, \mathrm{ref}} \tilde{a}_{l}^{F} \sin \left(\tilde{\rho}_{a, l}^{F}-\rho_{0}\right) \\
\overline{-2 \psi_{\mathrm{r} b} \tilde{\psi}_{\mathrm{r} b, l}^{F}} & =\psi_{\mathrm{r}, \mathrm{ref}} \tilde{b}_{l}^{F} \sin \left(\tilde{\rho}_{b, l}^{F}-\rho_{0}\right)
\end{aligned}
$$

Adding the terms on the right-hand sides of (86) and substituting into (84), we obtain

$$
\pi_{l}^{F}=\left|\sigma_{l}^{F}+\overline{\left(\tilde{\eta}_{a, l}^{F}\right)^{2}}+\overline{\left(\tilde{\eta}_{b, l}^{F}\right)^{2}}\right|, \quad F \in\{R, S, T\}
$$

where

$$
\begin{aligned}
& \sigma_{l}^{F} \triangleq \frac{\left(\tilde{a}_{l}^{F}\right)^{2}}{2}+\frac{\left(\tilde{b}_{l}^{F}\right)^{2}}{2}-\psi_{\mathrm{r}, \mathrm{ref}} \tilde{a}_{l}^{F} \sin \left(\tilde{\rho}_{a, l}^{F}-\rho_{0}\right) \\
& +\psi_{\mathrm{r}, \mathrm{ref}} \tilde{b}_{l}^{F} \sin \left(\tilde{\rho}_{b, l}^{F}-\rho_{0}\right), \quad l \in \mathcal{L}^{F}
\end{aligned}
$$

